\title{
OPTIMAL DISCONTINUOUS GALERKIN METHODS FOR WAVE PROPAGATION*
}

\author{
ERIC T. CHUNG $^{\dagger}$ AND BJÖRN ENGQUIST ${ }^{\ddagger}$
}

\begin{abstract}
We have developed and analyzed a new class of discontinuous Galerkin methods (DG) which can be seen as a compromise between standard DG and the finite element (FE) method in the way that it is explicit like standard DG and energy conserving like FE. In the literature there are many methods that achieve some of the goals of explicit time marching, unstructured grid, energy conservation, and optimal higher order accuracy, but as far as we know only our new algorithms satisfy all the conditions. We propose a new stability requirement for our DG. The stability analysis is based on the careful selection of the two FE spaces which verify the new stability condition. The convergence rate is optimal with respect to the order of the polynomials in the FE spaces. Moreover, the convergence is described by a series of numerical experiments.
\end{abstract}

Key words. discontinuous Galerkin, wave propagation, optimal rate of convergence

AMS subject classifications. $65 \mathrm{M} 12,65 \mathrm{M} 15,65 \mathrm{M} 60,78 \mathrm{M} 10$

DOI. $10.1137 / 050641193$

1. Introduction. Many applications involve the solution of wave equations. Examples are electromagnetic waves for radar and communication as well as acoustic and seismic wave propagation. Let $\Omega \subset \mathbb{R}^{2}$ be a two dimensional polygonal domain with outward normal vector $n$ and let $T>0$ be a fixed time. Given two positive constants $a_{1}>0, a_{2}>0$ and two given functions $F_{1}(x, t), F_{2}(x, t)$, we will consider, for $(x, t) \in \Omega \times(0, T)$, the following wave propagation problem: find a function $u(x, t)$ and a vector field $v(x, t) \in \mathbb{R}^{2}$ such that

$$
\begin{aligned}
a_{1} \frac{\partial u}{\partial t}+B v & =F_{1}, \\
a_{2} \frac{\partial v}{\partial t}-B^{*} u & =F_{2},
\end{aligned}
$$

where the two operators $B$ and $B^{*}$ satisfy

$$
\int_{\Omega_{0}}\left(B^{*} \phi\right) \psi d x-\int_{\Omega_{0}}(B \psi) \phi d x=\int_{\partial \Omega_{0}}(L \psi) \phi d \sigma
$$

for all subset $\Omega_{0} \subset \Omega$. Here $L$ is some operator depending on the two operators $B$, $B^{*}$ and the subdomain $\Omega_{0}$. We also denote by $L^{\perp}$ the operator such that $|\psi|^{2}=$ $|L \psi|^{2}+\left|L^{\perp} \psi\right|^{2}$. Assume that there is an operator $B^{\perp}$ such that $B B^{\perp} p=0$ for all $p$. Acting $\left(B^{\perp}\right)^{*}$ to $(1.2)$ and using $\left(B^{\perp}\right)^{*} B^{*}=0$, we have the following:

$$
\frac{\partial}{\partial t}\left(a_{2}\left(B^{\perp}\right)^{*} v\right)=\left(B^{\perp}\right)^{*} F_{2} .
$$

\footnotetext{
* Received by the editors September 26, 2005; accepted for publication (in revised form) April 6, 2006; published electronically November 3, 2006.

http://www.siam.org/journals/sinum/44-5/64119.html

$\dagger$ Applied and Computational Mathematics, California Institute of Technology, Pasadena, CA 91125 (tschung@acm.caltech.edu).

${ }^{\ddagger}$ Department of Mathematics, University of Texas at Austin, Austin, TX 78712 (engquist@math. utexas.edu).
} 
The condition (1.4) usually has important physical significance. For example, in the case of electromagnetic wave propagation (see (E) in the following), $v$ represents the electric field, $\left(B^{\perp}\right)^{*}$ is the divergence operator, and (1.4) is just the continuity equation expressing the conservation of charges. We supplement the system (1.1)-(1.2) with boundary condition

$$
L v=0 \quad \forall x \in \partial \Omega
$$

and initial conditions

$$
u(x, 0)=u_{0}(x) \quad \text { and } \quad v(x, 0)=v_{0}(x) \quad \forall x \in \Omega,
$$

where $u_{0}(x)$ and $v_{0}(x)$ are given. In particular, we are interested in the acoustic and the electromagnetic wave equations, which correspond to the following choice of $B$ and $B^{*}$ :

(A) Acoustic: $B v=-\nabla \cdot v, B^{*} u=\nabla u$, and $L v=v \cdot n$.

(E) Electromagnetic: $B v=\nabla \times v, B^{*} u=\nabla \times u$, and $L v=v \times n$.

Notice that, in (1.1)-(1.2), $u(x, t)$ is a function while $v(x, t)=\left(v^{1}(x, t), v^{2}(x, t)\right)$ is a vector having two components. So the operator $\nabla \times$ is defined as $\nabla \times v=\partial_{1} v^{2}-\partial_{2} v^{1}$ for any vector field $v$ and $\nabla \times u=\left(\partial_{2} u,-\partial_{1} u\right)$ for any function $u$. Here $v \times n=$ $v^{1} n_{2}-v^{2} n_{1}$ with $n=\left(n_{1}, n_{2}\right)$. In (A) and (E), we use $n$ to denote generically the unit normal vector of the corresponding subdomain which defines $L$ and $L^{\perp}$. Moreover, we have $L^{\perp} \psi=\psi \times n$ for (A) while $L^{\perp} \psi=\psi \cdot n$ for (E). Furthermore, we have $B^{\perp} p=\nabla \times p$ and $\left(B^{\perp}\right)^{*} p=\nabla \times p$ for $(\mathrm{A})$ while $B^{\perp} p=\nabla p$ and $\left(B^{\perp}\right)^{*} p=-\nabla \cdot p$ for (E). Wave propagation problems can be solved by partial differential equation (PDE) techniques, integral equation techniques, and asymptotic techniques. Among PDE techniques, finite difference (FD) method, finite volume (FV) method, finite element (FE) method, and discontinuous Galerkin (DG) method are the most popular choices. The FD method provides a simple way to solve wave propagation problems, but it is typically low order and applies only to structured grids. The FV method can be seen as a generalization of the FD method to unstructured grids, but it is still low order. The FE and DG methods provide high order solvers for the time dependent wave equations on unstructured grids.

Nédélec [15] introduces a curl-conforming FE method for solving Maxwell's equations. Geveci [8] proposed a mixed FE method for the scalar wave equation. The inversion of the mass matrix at each time step causes some possible drawback in the efficiency of those methods. Mass lumping techniques can be used to avoid solving linear systems. In Cohen and Monk [6], a mass lumping method for rectangular grids is developed. In Bécache, Joly, and Tsogka [1], a new class of mixed FE method, which is suitable for mass lumping, is developed for the scalar wave equation. Cohen, Joly, Torjman, and Roberts [5] design a mass lumping technique for triangular grids for polynomial order up to five. Discontinuous Galerkin methods provide explicit schemes in the sense that only block diagonal mass matrices have to be inverted. Hesthaven and Warburton [10] proposed a DG method based on upwind flux and Cockburn, Li, and Shu [4] proposed a DG method based on locally divergence free basis and upwind flux. While the schemes are successful, energy is not conserved due to the upwinding. Fezoui, Lanteri, Lohrengel, and Piperno [7] proposed a DG method based on central flux. This method preserves energy, but the convergence rate of the scheme is suboptimal. Recently, a new DG method has been developed for the wave equation in second order form; see Grote, Schneebeli, and Schötzau [9]. The method is also energy conserving 
DG

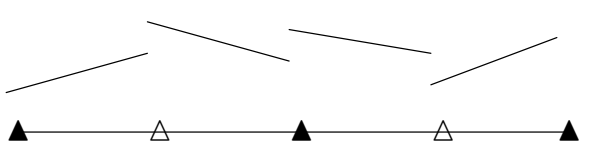

both $u$ and $v$ are discontinuous

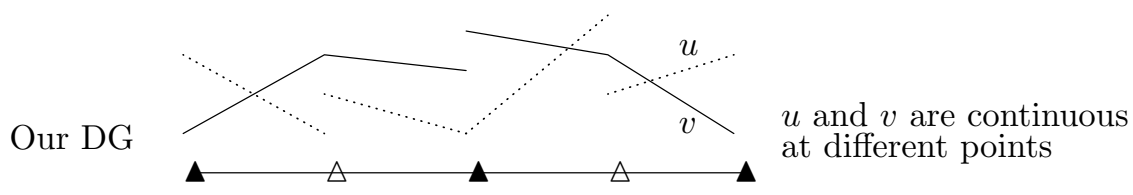

$\mathrm{FE}$

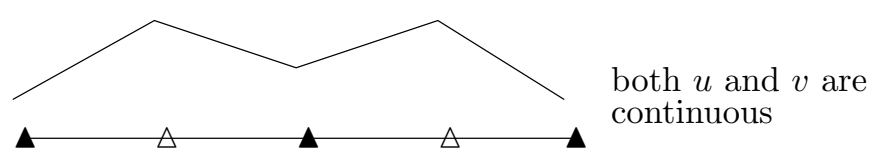

FIG. 1.1. Comparison among standard $D G$, our new $D G$, and FE methods.

in the sense of a newly defined energy. A space-time DG method has also been developed in Monk and Richter [14].

In this paper, we will develop and analyze a new class of DG methods which can be seen as a compromize between FE and DG methods. Our new DG method combines the advantages of FE and DG methods in the sense that it is both energy conserving and explicit. The idea is to use discontinuous functions with extra continuity. In the velocity-potential formulation of the scalar wave equation (A), we will add extra continuity to the velocity where the potential is discontinuous and add extra continuity to the potential where the velocity is discontinuous. For Maxwell's equations (E), a similar idea can be applied to the electric and magnetic fields. As a result, the flux integrals are evaluated exactly, which is the basis of energy conservation. However, the addition of the extra continuity cannot be done arbitrarily due to stability concerns. It has to be done in such a way that some inf-sup conditions are satisfied. In Figure 1.1, we illustrate this idea in one space dimension. For standard DG, both unknown functions $u$ and $v$, which are velocity and potential for scalar wave equation and are electric and magnetic fields for Maxwell's equations, are discontinuous at cell boundaries. For FE methods, both $u$ and $v$ are continuous. For our new DG, the two functions are continuous at different points.

Yee's scheme [16] has been a very popular numerical method for computational electromagnetics. It is a second order central FD method on structured grids. The success of the scheme is due to the use of a staggered grid. Our new DG method is a FE method on staggered grids and can be seen as a higher generalization of Yee's scheme on unstructured grids. In particular, in one space dimension, our new DG method with piecewise constant approximation is the same as Yee's scheme. In two space dimensions, our new DG method in the lowest order is some averaged version of Yee's scheme.

The rest of the paper is organized as follows. In section 2, we will introduce the new FE spaces and prove the corresponding unisolvence and interpolation error estimates. The new DG is then derived in section 3. In section 4, under the assumption of some inf-sup conditions, the stability and convergence of the method are proved. The inf-sup conditions are then verified in section 5. Furthermore, some numerical experiments are presented in section 6 . The paper ends with a conclusion.

Remark. We consider only two space dimensions in this paper. For three space dimensions, a careful choice of the two FE spaces $U_{h}$ and $V_{h}$ that verify (3.1) and 
(3.2) as well as the two inf-sup conditions (4.1)-(4.2) are required. This work will be developed in a forthcoming paper.

2. FE spaces. Assume the domain $\Omega$ is triangulated by a family of triangles $\mathcal{T}$ so that $\Omega=\cup\{\tau \mid \tau \in \mathcal{T}\}$. Let $\tau \in \mathcal{T}$. We define $h_{\tau}$ as the diameter of $\tau$ and $\rho_{\tau}$ as the supremum of the diameters of the circles inscribed in $\tau$. The mesh size $h$ is defined as $h=\max _{\tau \in \mathcal{T}} h_{\tau}$. We will assume the set of triangles $\mathcal{T}$ forms a regular family of triangulation of $\Omega$ so that there exist a uniform constant $K$ independent of the mesh size such that [3]

$$
h_{\tau} \leq K \rho_{\tau} \quad \forall \tau \in \mathcal{T} .
$$

In addition, we will assume the triangulation satisfies the inverse assumption [3].

Let $\mathcal{E}$ be the set of all edges and let $\mathcal{E}^{0} \subset \mathcal{E}$ be the set of all interior edges of the triangles in $\mathcal{T}$. The length of $\sigma \in \mathcal{E}$ will be denoted by $h_{\sigma}$. We also denote by $\mathcal{N}$ the set of all interior nodes of the triangles in $\mathcal{T}$. Here, by interior edge and interior node, we mean any edge and node that does not lie on the boundary $\partial \Omega$. Let $\nu \in \mathcal{N}$. We define

$$
\mathcal{S}(\nu)=\cup\{\tau \in \mathcal{T} \mid \nu \in \tau\}
$$

That is, $\mathcal{S}(\nu)$ is the union of all triangles having vertex $\nu$. We will assume the triangulation of $\Omega$ satisfies the following condition.

Assumption on triangulation: There exists a subset $\mathcal{N}_{1} \subset \mathcal{N}$ such that

(A1) $\Omega=\cup\left\{\mathcal{S}(\nu) \mid \nu \in \mathcal{N}_{1}\right\}$.

(A2) $\mathcal{S}\left(\nu_{i}\right) \cap \mathcal{S}\left(\nu_{j}\right) \in \mathcal{E}^{0}$ for all distinct $\nu_{i}, \nu_{j} \in \mathcal{N}_{1}$.

Let $\nu \in \mathcal{N}_{1}$. We define

$$
\mathcal{E}_{u}(\nu)=\{\sigma \in \mathcal{E} \mid \nu \in \sigma\} .
$$

That is, $\mathcal{E}_{u}(\nu)$ is the set of all edges that have $\nu$ as one of their endpoints. We further define

$$
\mathcal{E}_{u}=\cup\left\{\mathcal{E}_{u}(\nu) \mid \nu \in \mathcal{N}_{1}\right\} \quad \text { and } \quad \mathcal{E}_{v}=\mathcal{E} \backslash \mathcal{E}_{u} .
$$

Notice that $\mathcal{E}_{u}$ contains only interior edges since one of the endpoints of edges in $\mathcal{E}_{u}$ has a vertex from $\mathcal{N}_{1}$. On the other hand, $\mathcal{E}_{v}$ has both interior and boundary edges. So, we also define $\mathcal{E}_{v}^{0}=\mathcal{E}_{v} \cap \mathcal{E}^{0}$ which contains elements from $\mathcal{E}_{v}$ that are interior edges. Notice that we have $\mathcal{E}_{v} \backslash \mathcal{E}_{v}^{0}=\mathcal{E} \cap \partial \Omega$. Furthermore, for $\sigma \in \mathcal{E}_{v}^{0}$, we will let $\mathcal{R}(\sigma)$ be the union of the two triangles sharing the same edge $\sigma$. For $\sigma \in \mathcal{E}_{v} \backslash \mathcal{E}_{v}^{0}$, we will let $\mathcal{R}(\sigma)$ be the only triangle having the edge $\sigma$.

In practice, triangulations that satisfy assumptions (A1)-(A2) are not difficult to construct. In Figure 2.1, we illustrate how this kind of triangulation is generated. First, the domain $\Omega$ is triangulated by a family of triangles, called $\tilde{\mathcal{T}}$. Each triangle in this family is then subdivided into three subtriangles by connecting a point inside the triangle with its three vertices. Then we define the union of all these subtriangles to be our triangulation $\mathcal{T}$. Each triangle in $\tilde{\mathcal{T}}$ corresponds to an $\mathcal{S}(\nu)$ for some $\nu$ inside the triangle. In Figure 2.1, we show two of the triangles, enclosed by solid lines, in this family $\tilde{\mathcal{T}}$. This corresponds to 6 triangles in the triangulation $\mathcal{T}$. The dotted lines represent edges in the set $\mathcal{E}_{u}$ while solid lines represent edges in the set $\mathcal{E}_{v}$.

Lemma 2.1. Each $\tau \in \mathcal{T}$ has exactly two edges that belong to $\mathcal{E}_{u}$. 


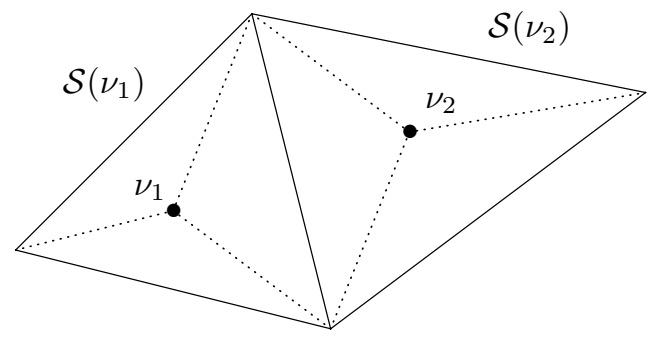

FiG. 2.1. Triangulation.

Proof. First of all, $\tau$ has at least one interior vertex. We will show that there is exactly one vertex of $\tau$ that belongs to $\mathcal{N}_{1}$. If none of the three vertices of $\tau$ belong to $\mathcal{N}_{1}$, then $\tau^{0} \cap \mathcal{S}(\nu)$ is an empty set for all $\nu \in \mathcal{N}_{1}$, where $\tau^{0}$ is the interior of $\tau$. Then, $\cup\left\{\mathcal{S}(\nu) \mid \nu \in \mathcal{N}_{1}\right\} \cap \tau^{0}$ is an empty set. So, $\cup\left\{\mathcal{S}(\nu) \mid \nu \in \mathcal{N}_{1}\right\} \neq \Omega$, which violates assumption (A1). If $\tau$ has two vertices, $\nu_{i}$ and $\nu_{j}$, that belong to $\mathcal{N}_{1}$, then $\mathcal{S}\left(\nu_{i}\right) \cap \mathcal{S}\left(\nu_{j}\right)$ contains $\tau$. So, it violates assumption (A2). The case that $\tau$ has all vertices belonging to $\mathcal{N}_{1}$ can be discussed in the same way. In conclusion, $\tau$ has exactly one vertex which belongs to $\mathcal{N}_{1}$. So, by the definition of $\mathcal{E}_{u}$, the two edges having the vertex in $\mathcal{N}_{1}$ belong to $\mathcal{E}_{u}$.

Given $\tau \in \mathcal{T}$, we will denote by $\nu(\tau)_{1}, \nu(\tau)_{2}$, and $\nu(\tau)_{3}$ the three vertices of $\tau$. Moreover, $\nu(\tau)_{1}$ is the vertex that is one of the endpoints of the two edges of $\tau$ that belong to $\mathcal{E}_{u}$. Then $\nu(\tau)_{2}$ and $\nu(\tau)_{3}$ are named in a counterclockwise direction. In addition, $\lambda_{\tau, 1}(x), \lambda_{\tau, 2}(x)$, and $\lambda_{\tau, 3}(x)$ are the barycentric coordinates on $\tau$ with respect to the three vertices $\nu(\tau)_{1}, \nu(\tau)_{2}$, and $\nu(\tau)_{3}$.

Now, we will discuss the FE spaces. Let $k \geq 0$ be a nonnegative integer. Let $\tau \in \mathcal{T}$. We define $P^{k}(\tau)$ as the space of polynomials of degree less than or equal to $k$ on $\tau$. We also define

$$
R^{k}(\tau)=P^{k}(\tau) \oplus \tilde{P}^{k+1}(\tau),
$$

where $\tilde{P}^{k+1}(\tau)$ is the space of homogeneous polynomials of degree $k+1$ on $\tau$ in the two variables $\lambda_{\tau, 2}$ and $\lambda_{\tau, 3}$ such that the sum of the coefficients of $\lambda_{\tau, 2}^{k+1}$ and $\lambda_{\tau, 3}^{k+1}$ is equal to zero. That is, any function in $\tilde{P}^{k+1}(\tau)$ can be written as $\sum_{i+j=k+1, i \geq 0, j \geq 0} a_{i, j} \lambda_{\tau, 2}^{i} \lambda_{\tau, 3}^{j}$ such that $a_{k+1,0}+a_{0, k+1}=0$. Now, we define

$$
U_{h}=\left\{\phi|\phi|_{\tau} \in R^{k}(\tau) ; \phi \text { is continuous at the } k+1 \text { Gaussian points of } \sigma \forall \sigma \in \mathcal{E}_{u}\right\} .
$$

For any edge $\sigma$, we use $P^{k}(\sigma)$ to represent the space of one dimensional polynomials of degree less than or equal to $k$ on $\sigma$. We define the following degrees of freedom:

(UD1) For each edge $\sigma \in \mathcal{E}_{u}$, we have

$$
\int_{\sigma} \phi p_{k} d \sigma
$$

for all $p_{k} \in P^{k}(\sigma)$.

(UD2) For each triangle $\tau \in \mathcal{T}$, we have

$$
\int_{\tau} \phi p_{k-1} d x
$$

for all $p_{k-1} \in P^{k-1}(\tau)($ for $k \geq 1)$. 
Notice that (UD1) is equivalent to $\phi\left(\alpha_{i}\right)$ where $\alpha_{i}$, for $i=1,2, \ldots, k+1$, are the $k+1$ Gaussian points of $\sigma$. For a smooth function $\phi$, we will define $\mathcal{I}_{u} \phi \in U_{h}$ by the following degrees of freedom:

(U1) For each edge $\sigma \in \mathcal{E}_{u}$, we have

$$
\int_{\sigma}\left(\mathcal{I}_{u} \phi-\phi\right) p_{k}=0
$$

for all $p_{k} \in P^{k}(\sigma)$.

(U2) For each triangle $\tau \in \mathcal{T}$, we have

$$
\int_{\tau}\left(\mathcal{I}_{u} \phi-\phi\right) p_{k-1} d x=0
$$

for all $p_{k-1} \in P^{k-1}(\tau)$ (for $\left.k \geq 1\right)$.

TheOREM 2.2. Let $\mathcal{I}_{u}: \Pi_{\nu \in \mathcal{N}_{1}} W^{k+1, p}(\mathcal{S}(\nu)) \rightarrow U_{h}$. Then $\mathcal{I}_{u}$ is uniquely determined by (U1)-(U2). Moreover,

$$
\left|\phi-\mathcal{I}_{u} \phi\right|_{W^{m, p}(\mathcal{S}(\nu))} \leq K h^{k+1-m}|\phi|_{W^{k+1, p}(\mathcal{S}(\nu))} .
$$

Proof. Notice that $\operatorname{dim}\left(P^{k}\right)=\frac{1}{2}(k+1)(k+2)$. Then (UD1) gives $(k+1)\left|\mathcal{E}_{u}\right|$ conditions while (UD2) gives $\frac{1}{2} k(k+1)|\mathcal{T}|$ conditions where $|\mathcal{S}|$ is the number of elements in the set $\mathcal{S}$. Notice that $|\mathcal{S}(\nu)|=\left|\mathcal{E}_{u}(\nu)\right|$ for all $\nu \in \mathcal{N}_{1}$. So, by the assumption (A1)(A2) and the definition of $\mathcal{E}_{u}$, we have $|\mathcal{T}|=\sum_{\nu \in \mathcal{N}_{1}}|\mathcal{S}(\nu)|=\sum_{\nu \in \mathcal{N}_{1}}\left|\mathcal{E}_{u}(\nu)\right|=\left|\mathcal{E}_{u}\right|$. So, the total number of degrees of freedom defined by (UD1)-(UD2) is $\frac{1}{2}(k+1)(k+$ $2)|\mathcal{T}|$. Next, we will find the $\operatorname{dim}\left(U_{h}\right)$. Notice that $\operatorname{dim}\left(\tilde{P}^{k+1}(\tau)\right)=k+1$. So, we have $\operatorname{dim}\left(U_{h}\right)=\frac{1}{2}(k+1)(k+2)|\mathcal{T}|+(k+1)|\mathcal{T}|-(k+1)\left|\mathcal{E}_{u}\right|$, where the subtraction of the third term is due to the continuity condition imposed on the $k+1$ Gaussian points of each edge in $\mathcal{E}_{u}$. Since $|\mathcal{T}|=\left|\mathcal{E}_{u}\right|$, we have $\operatorname{dim}\left(U_{h}\right)=\frac{1}{2}(k+1)(k+2)|\mathcal{T}|$, which is equal to the number of degrees of freedom defined by (UD1)-(UD2).

Next, we will show $\mathcal{I}_{u} \phi=0$ if $\phi=0$. Let $\tau \in \mathcal{T}$. Then the degree of freedom (UD1) implies that $\mathcal{I}_{u} \phi$ is zero at the $k+1$ Gaussian points of the two edges of $\tau$ that belong to $\mathcal{E}_{u}$. More precisely, we denote by $\alpha_{j}(j=1,2, \ldots, k+1)$ the $k+1$ Gaussian points of the edge of $\tau$ having endpoints $\nu(\tau)_{1}$ and $\nu(\tau)_{2}$. Then we define real numbers $w_{j}(j=1,2, \ldots, k+1)$ such that $0<w_{1}<w_{2}<\cdots<w_{k+1}<1$ and $\alpha_{j}=\left(1-w_{j}\right) \nu(\tau)_{1}+w_{j} \nu(\tau)_{2}$. Moreover, we denote by $\beta_{j}(j=1,2, \ldots, k+1)$ the $k+1$ Gaussian points of the edge of $\tau$ having endpoints $\nu(\tau)_{1}$ and $\nu(\tau)_{3}$. Then the real numbers $w_{j}$ also satisfy $\beta_{j}=\left(1-w_{j}\right) \nu(\tau)_{1}+w_{j} \nu(\tau)_{3}$. So, we have

$$
\mathcal{I}_{u} \phi=c \Pi_{j=1}^{k+1}\left(\lambda_{\tau, 2}-w_{j}\right)+c \Pi_{j=1}^{k+1}\left(\lambda_{\tau, 3}-w_{j}\right)-c(-1)^{k+1} \Pi_{j=1}^{k+1} w_{j}+\lambda_{\tau, 2} \lambda_{\tau, 3} q_{k-1}
$$

for some $q_{k-1} \in P^{k-1}(\tau)$. By the definition of $R^{k}(\tau)$, the sum of the coefficients of $\lambda_{\tau, 2}^{k+1}$ and $\lambda_{\tau, 3}^{k+1}$ is zero. So, we have $c=0$. Using (UD2), we have $\int_{\tau} \lambda_{\tau, 2} \lambda_{\tau, 3} q_{k-1}^{2} d x=0$. Since $\lambda_{\tau, 2} \lambda_{\tau, 3}>0$ in the interior of $\tau$, we have $q_{k-1}=0$. Hence, $\mathcal{I}_{u} \phi=0$.

Now, we will prove (2.5). Let $\tau \in \mathcal{T}$ and let $p_{k} \in P^{k}(\tau)$. It suffices to show that $\mathcal{I}_{u}$ preserves polynomials. By (U1), $\mathcal{I}_{u} p_{k}-p_{k}$ is zero at the $k+1$ Gaussian points of the two edges that belong to $\mathcal{E}_{u}$. So,

$$
\begin{aligned}
\mathcal{I}_{u} p_{k}-p_{k}= & b \Pi_{j=1}^{k+1}\left(\lambda_{\tau, 2}-w_{j}\right)+b \Pi_{j=1}^{k+1}\left(\lambda_{\tau, 3}-w_{j}\right) \\
& -b(-1)^{k+1} \Pi_{j=1}^{k+1} w_{j}+\lambda_{\tau, 2} \lambda_{\tau, 3} r_{k-1}
\end{aligned}
$$


for some constant $d$ and $r_{k-1} \in P^{k-1}(\tau)$. Since $\mathcal{I}_{u} p_{k} \in R^{k}(\tau)$, we have $b=0$. Using (U2), we have $r_{k-1}=0$. Hence, $\mathcal{I}_{u} p_{k}=p_{k}$.

We define

$$
V_{h}=\left\{\psi|\psi|_{\tau} \in P^{k}(\tau)^{2} ; L \psi \text { is continuous along } \sigma \forall \sigma \in \mathcal{E}_{v} ; L \psi=0 \text { on } \partial \Omega\right\} .
$$

We also define the following degrees of freedom:

(VD1) For each triangle $\tau \in \mathcal{T}$, we have

$$
\int_{\tau}\left(L^{\perp} \psi\right) p_{k} d x \quad \forall p_{k} \in P^{k}(\tau)
$$

(VD2) For each triangle $\tau \in \mathcal{T}$, we have

$$
\int_{\tau}(L \psi) \lambda_{\tau, 1} p_{k-1} d x \quad \forall p_{k-1} \in P^{k-1}(\tau) .
$$

(VD3) For each edge $\sigma \in \mathcal{E}_{v}^{0}$, we have

$$
\int_{\sigma}(L \psi) q_{k} d \sigma \quad \forall q_{k} \in P^{k}(\sigma)
$$

Furthermore, for a smooth vector field $\psi$, we define $\mathcal{I}_{v} \psi$ as the corresponding interpolation operator by the following degrees of freedom:

(V1) For each triangle $\tau \in \mathcal{T}$, we have

$$
\int_{\tau} L^{\perp}\left(\mathcal{I}_{v} \psi-\psi\right) p_{k} d x=0 \quad \forall p_{k} \in P^{k}(\tau)
$$

(V2) For each triangle $\tau \in \mathcal{T}$, we have

$$
\int_{\tau} L\left(\mathcal{I}_{v} \psi-\psi\right) \lambda_{\tau, 1} p_{k-1} d x=0 \quad \forall p_{k-1} \in P^{k-1}(\tau) .
$$

(V3) For each edge $\sigma \in \mathcal{E}_{v}^{0}$, we have

$$
\int_{\sigma} L\left(\mathcal{I}_{v} \psi-\psi\right) q_{k} d \sigma=0 \quad \forall q_{k} \in P^{k}(\sigma) .
$$

TheOREM 2.3. Let $\mathcal{I}_{v}: \Pi_{\sigma \in \mathcal{E}_{v}^{0}} W^{k+1, p}(\mathcal{R}(\sigma))^{2} \rightarrow V_{h}$. Then $\mathcal{I}_{v}$ is uniquely determined by (V1), (V2), and (V3). Moreover,

$$
\left|\psi-\mathcal{I}_{v} \psi\right|_{W^{m, p}(\mathcal{R}(\sigma))^{2}} \leq K h^{k+1-m}|\psi|_{W^{k+1, p}(\mathcal{R}(\sigma))^{2}} .
$$

Proof. First of all, the number of degrees of freedom defined by (VD1), (VD2), and (VD3) are $\frac{1}{2}(k+1)(k+2)|\mathcal{T}|, \frac{1}{2} k(k+1)|\mathcal{T}|$, and $(k+1)\left|\mathcal{E}_{v}^{0}\right|$, respectively. Also, the dimension of $V_{h}$ is given by

$$
\operatorname{dim}\left(V_{h}\right)=2 \frac{1}{2}(k+1)(k+2)|\mathcal{T}|-(k+1)\left|\mathcal{E}_{v}^{0}\right|-(k+1)\left|\mathcal{E}_{v} \backslash \mathcal{E}_{v}^{0}\right|,
$$

where the second term on the right-hand side is due to the continuity condition that $L \psi$ is continuous on $\sigma$ for all $\sigma \in \mathcal{E}_{v}^{0}$, and the third term on the right-hand side is due to the boundary condition $L \psi=0$ on $\partial \Omega$. Notice that $|\mathcal{T}|=2\left|\mathcal{E}_{v}^{0}\right|+\left|\mathcal{E}_{v} \backslash \mathcal{E}_{v}^{0}\right|$. 


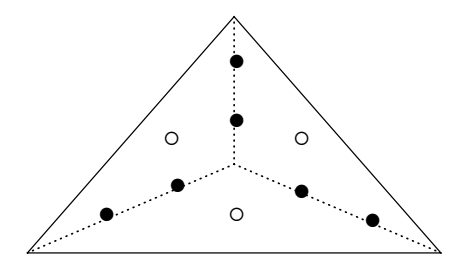

FIG. 2.2. $\mathcal{S}(\nu)$ for $k=1$.

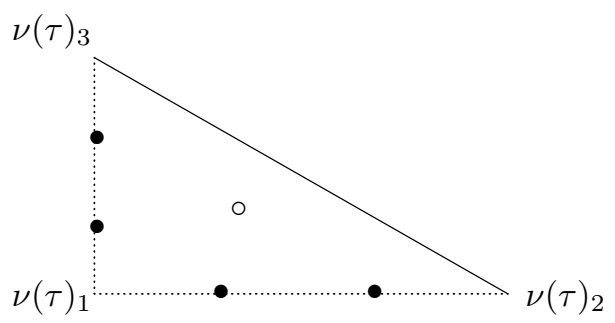

FIG. 2.3. A single triangle for $k=1$.

Now, a direct calculation shows that $\operatorname{dim}\left(V_{h}\right)$ is equal to the total number of degrees of freedom defined by (VD1), (VD2), and (VD3).

Now, we will show $\mathcal{I}_{v} \psi=0$ if $\psi=0$. First, (VD1) implies that $L^{\perp}\left(\mathcal{I}_{v} \psi\right)=0$ on each $\tau \in \mathcal{T}$. Using (VD3), we have $L\left(\mathcal{I}_{v} \psi\right)=0$ on each $\sigma \in \mathcal{E}_{v}^{0}$. So, on each $\tau \in \mathcal{T}$, we have $L\left(\mathcal{I}_{v} \psi\right)=\lambda_{\tau, 1} q_{k-1}$ for some $q_{k-1} \in P^{k-1}(\tau)$. Indeed, we can write

$$
L\left(\mathcal{I}_{v} \psi\right)=a+\sum_{j=1}^{k}\left(b_{j} \lambda_{\tau, 1}^{j}+c_{j} \lambda_{\tau, 2}^{j}\right) .
$$

Since $\left.L\left(\mathcal{I}_{v} \psi\right)\right|_{\sigma}=0$, we have $a=0, c_{j}=0$, and $L\left(\mathcal{I}_{v} \psi\right)=\sum_{j=1}^{k} b_{j} \lambda_{\tau, 1}^{j}$. Applying (VD2), we have $L\left(\mathcal{I}_{v} \psi\right)=0$ on each $\tau \in \mathcal{T}$. Since $\left|\mathcal{I}_{v} \psi\right|^{2}=\left|L^{\perp}\left(\mathcal{I}_{v} \psi\right)\right|^{2}+\left|L\left(\mathcal{I}_{v} \psi\right)\right|^{2}$, we have $\mathcal{I}_{v} \psi=0$.

The estimate (2.6) follows from the fact that the operator $\mathcal{I}_{v}$ preserves polynomials of degree $k$.

Let us consider an example for $k=1$. For $U_{h}$, the degrees of freedom are the two Gaussian points on each edge belonging to $\mathcal{E}_{u}$ and the cell center. In Figure 2.2, we illustrate three triangles in the triangulation which corresponds to an $\mathcal{S}(\nu)$ for some $\nu \in \mathcal{N}_{1}$. The dotted lines denote edges from the set $\mathcal{E}_{u}$ while solid lines denote edges from the set $\mathcal{E}_{v}$. The solid dots denote the continuity points defined by (UD1), which are the two Gaussian points of the edges in $\mathcal{E}_{u}$. The circle in each triangle represents the degree of freedom defined by (UD2). In Figure 2.3, we show the degrees of freedom on a single triangle. Any function $\phi \in R^{k}(\tau)$ can be expressed as

$$
\phi=a+b \lambda_{\tau, 2}+c \lambda_{\tau, 3}+d\left(\lambda_{\tau, 2}^{2}-\lambda_{\tau, 3}^{2}\right)+e \lambda_{\tau, 2} \lambda_{\tau, 3} .
$$

For $V_{h}, L \psi$ is defined as a linear function which is continuous on the edge $\sigma$ while $L^{\perp}$ is defined as a linear function on each triangle with no continuity requirement. In Figure 2.4, we illustrate an $\mathcal{R}(\sigma)$ for some $\sigma \in \mathcal{E}_{v}^{0}$, where $\sigma$ is represented by the solid line. We represent the degrees of freedom of $L \psi$ by solid dots and the degrees of freedom of $L^{\perp} \psi$ by circles. 


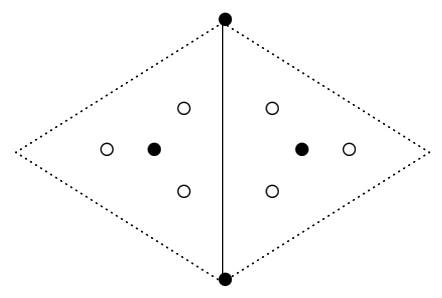

FIG. 2.4. $\mathcal{R}(\sigma)$ for $k=1$.

3. The new scheme. In this section, we will derive the new discontinuous Galerkin method for the wave propagation problem (1.1)-(1.2). Multiplying both sides of (1.1) by $\phi$, integrating the resulting equation on $\mathcal{S}(\nu)$, and using (1.3) yields

$$
a_{1} \int_{\mathcal{S}(\nu)} \frac{\partial u}{\partial t} \phi d x+\int_{\mathcal{S}(\nu)}\left(B^{*} \phi\right) v d x-\int_{\partial \mathcal{S}(\nu)}(L v) \phi d \sigma=\int_{\mathcal{S}(\nu)} F_{1} \phi d x .
$$

Summing over all $\nu \in \mathcal{N}_{1}$,

$$
a_{1} \int_{\Omega} \frac{\partial u}{\partial t} \phi d x+\int_{\Omega}\left(B^{*} \phi\right) v d x-\sum_{\nu \in \mathcal{N}_{1}} \int_{\partial \mathcal{S}(\nu)}(L v) \phi d \sigma=\int_{\Omega} F_{1} \phi d x
$$

If $L v$ is continuous along each $\sigma \in \mathcal{E}_{v}^{0}$ and $L v$ is zero along each edge in $\mathcal{E}_{v} \backslash \mathcal{E}_{v}^{0}$, then

$$
\sum_{\nu \in \mathcal{N}_{1}} \int_{\partial \mathcal{S}(\nu)}(L v) \phi d \sigma=\sum_{\sigma \in \mathcal{E}_{v}^{0}} \int_{\sigma}(L v)[\phi] d \sigma
$$

where $[\phi]=\phi^{+}-\phi^{-}$is the jump of $\phi$ along $\sigma$. Similarly, multiplying both sides of (1.2) by $\psi$, integrating the resulting equation on $\mathcal{R}(\sigma)$, and using (1.3) yields

$$
a_{2} \int_{\mathcal{R}(\sigma)} \frac{\partial v}{\partial t} \psi d x-\int_{\mathcal{R}(\sigma)}(B \psi) u d x-\int_{\partial \mathcal{R}(\sigma)}(L \psi) u d \sigma=\int_{\mathcal{R}(\sigma)} F_{2} \psi d x .
$$

Summing for all $\sigma \in \mathcal{E}_{v}$,

$$
a_{2} \int_{\Omega} \frac{\partial v}{\partial t} \psi d x-\int_{\Omega}(B \psi) u d x-\sum_{\sigma \in \mathcal{E}_{v}} \int_{\partial \mathcal{R}(\sigma)}(L \psi) u d \sigma=\int_{\Omega} F_{2} \psi d x .
$$

Now if $L \psi$ is a polynomial of degree $k$ and $u$ is a $(k+1)$ th degree polynomial which is continuous at the $k+1$ Gaussian points of $\sigma \in \mathcal{E}_{u}$, then

$$
\sum_{\sigma \in \mathcal{E}_{v}} \int_{\partial \mathcal{R}(\sigma)}(L \psi) u d \sigma=\sum_{\sigma \in \mathcal{E}_{u}} \int_{\sigma}[L \psi] u d \sigma
$$

where $[L \psi]$ denotes the jump of $L \psi$ along $\sigma$. Then, the new discontinuous Galerkin method is defined as follows.

The new discontinuous Galerkin method: Find $u_{h} \in U_{h}$ and $v_{h} \in V_{h}$ such that

$$
\begin{aligned}
& a_{1} \int_{\Omega} \frac{\partial u_{h}}{\partial t} \phi d x+B_{h}\left(v_{h}, \phi\right)=\int_{\Omega} F_{1} \phi d x, \\
& a_{2} \int_{\Omega} \frac{\partial v_{h}}{\partial t} \psi d x-B_{h}^{*}\left(u_{h}, \psi\right)=\int_{\Omega} F_{2} \psi d x
\end{aligned}
$$


for all $\phi \in U_{h}$ and $\psi \in V_{h}$, where

$$
\begin{aligned}
& B_{h}\left(v_{h}, \phi\right)=\int_{\Omega}\left(B^{*} \phi\right) v_{h} d x-\sum_{\sigma \in \mathcal{E}_{v}^{0}} \int_{\sigma}\left(L v_{h}\right)[\phi] d \sigma, \\
& B_{h}^{*}\left(u_{h}, \psi\right)=\int_{\Omega}(B \psi) u_{h} d x+\sum_{\sigma \in \mathcal{E}_{u}} \int_{\sigma}[L \psi] u_{h} d \sigma .
\end{aligned}
$$

The initial conditions $u_{h}(0)$ and $v_{h}(0)$ will be defined as $u_{h}(0)=\mathcal{I}_{u} u_{0}$ and $v_{h}(0)=$ $\mathcal{I}_{v} v_{0}$. We remark here that we define the spaces $U_{h}$ and $V_{h}$ so that (3.1) and (3.2) are valid. Furthermore, we define the discrete derivative operators $B_{h}$ and $B_{h}^{*}$ by

$$
\begin{aligned}
& \left\langle B_{h} \psi, \phi\right\rangle=B_{h}(\psi, \phi) \quad \forall \phi \in U_{h}, \\
& \left\langle B_{h}^{*} \phi, \psi\right\rangle=B_{h}^{*}(\phi, \psi) \quad \forall \psi \in V_{h} .
\end{aligned}
$$

The two operators $B_{h}$ and $B_{h}^{*}$ are the discrete analogue of the two derivative operators $B$ and $B^{*}$.

Lemma 3.1. For all $\phi \in U_{h}$ and $\psi \in V_{h}$, we have

$$
B_{h}(\psi, \phi)=B_{h}^{*}(\phi, \psi) \text {. }
$$

Proof. Let $\phi \in U_{h}$ and $\psi \in V_{h}$. Then, by the definition of $B_{h}$ and (3.1),

$$
\begin{aligned}
B_{h}(\psi, \phi) & =\int_{\Omega}\left(B^{*} \phi\right) v_{h} d x-\sum_{\sigma \in \mathcal{E}_{v}^{0}} \int_{\sigma}\left(L v_{h}\right)[\phi] d \sigma \\
& =\int_{\Omega}\left(B^{*} \phi\right) \psi d x-\sum_{\nu \in \mathcal{N}_{1}} \int_{\partial \mathcal{S}(\nu)}(L \psi) \phi d \sigma \\
& =\sum_{\nu \in \mathcal{N}_{1}}\left\{\int_{\mathcal{S}(\nu)}\left(B^{*} \phi\right) \psi d x-\int_{\partial \mathcal{S}(\nu)}(L \psi) \phi d \sigma\right\}
\end{aligned}
$$

Using integration by parts on each triangle,

$$
B_{h}(\psi, \phi)=\sum_{\nu \in \mathcal{N}_{1}}\left\{\int_{\mathcal{S}(\nu)} \phi(B \psi) d x+\sum_{\sigma \in \mathcal{E}_{u}(\nu)} \int_{\sigma}[L \psi] u d \sigma\right\}=B_{h}^{*}(\phi, \psi) .
$$

This completes the proof.

We define the discrete $L^{2}$ norms and $H^{1}$ norms in the following ways. For all $\phi \in U_{h}$, we define

$$
\begin{aligned}
\|\phi\|_{W}^{2} & =\int_{\Omega} \phi^{2} d x+\sum_{\sigma \in \mathcal{E}_{u}}\left(h_{\sigma}\right)^{2} \sum_{j=1}^{k+1} \phi\left(\alpha_{j}\right)^{2}, \\
\|\phi\|_{Z}^{2} & =\int_{\Omega}\left(B^{*} \phi\right)^{2} d x+\sum_{\sigma \in \mathcal{E}_{v}} h_{\sigma}^{-1} \int_{\sigma}[\phi]^{2} d \sigma .
\end{aligned}
$$

For all $\psi \in V_{h}$, we define

$$
\begin{aligned}
\|\psi\|_{W^{\prime}}^{2} & =\int_{\Omega} \psi^{2} d x+\sum_{\sigma \in \mathcal{E}_{v}} h_{\sigma} \int_{\sigma}(L \psi)^{2} d \sigma \\
\|\psi\|_{Z^{\prime}}^{2} & =\int_{\Omega}(B \psi)^{2} d x+\sum_{\sigma \in \mathcal{E}_{u}}\left(h_{\sigma}\right)^{-1} \int_{\sigma}[L \psi]^{2} d \sigma .
\end{aligned}
$$


With these definitions, we have the following continuity conditions for all $\phi \in U_{h}$ and $\psi \in V_{h}$ :

$$
\begin{aligned}
\left|B_{h}(\psi, \phi)\right| & \leq K\|\psi\|_{W^{\prime}}\|\phi\|_{Z}, \\
\left|B_{h}(\psi, \phi)\right| & \leq K\|\psi\|_{Z^{\prime}}\|\phi\|_{W} .
\end{aligned}
$$

Moreover, we have, in $U_{h}$, the norm $\|\phi\|_{W}$ is equivalent to the standard $L^{2}$ norm $\|\phi\|$, while in $V_{h}$, the norm $\|\psi\|_{W^{\prime}}$ is equivalent to the standard $L^{2}$ norm $\|\psi\|$. That is, there are two uniform constants $K_{1}$ and $K_{2}$ such that

$$
\begin{aligned}
& K_{1}\|\phi\| \leq\|\phi\|_{W} \leq K_{2}\|\phi\| \forall \phi \in U_{h}, \\
& K_{1}\|\psi\| \leq\|\psi\|_{W^{\prime}} \leq K_{2}\|\psi\| \quad \forall \psi \in V_{h} .
\end{aligned}
$$

Now, we will prove the following interpolation error estimates. The first one is the interpolation error in the discrete $L^{2}$ norms.

Lemma 3.2. Assume $(u, v) \in W^{k+1, \infty}(\Omega)^{3}$. Then for any integer $m$ with $1 \leq$ $m \leq k+1$,

$$
\left\|u-\mathcal{I}_{u} u\right\|_{W} \leq K h^{m}|u|_{W^{m, \infty}(\Omega)}, \quad\left\|v-\mathcal{I}_{v} v\right\|_{W^{\prime}} \leq K h^{m}|v|_{W^{m, \infty}(\Omega)^{2}} .
$$

Proof. By the definition of $W$-norm and $W^{\prime}$-norm,

$$
\left\|u-\mathcal{I}_{u} u\right\|_{W} \leq K\left\|u-\mathcal{I}_{u} u\right\|_{L^{\infty}(\Omega)}, \quad\left\|v-\mathcal{I}_{v} v\right\|_{W^{\prime}} \leq K\left\|v-\mathcal{I}_{v} v\right\|_{L^{\infty}(\Omega)^{2}} .
$$

The proof is complete by using (2.5) and (2.6).

The second one is the interpolation error in the discrete $H^{1}$ norms.

Lemma 3.3. Assume $(u, v) \in H^{k+1}(\Omega)^{3}$. Then for any integer $m$ with $1 \leq m \leq k$,

$$
\left\|u-\mathcal{I}_{u} u\right\|_{Z} \leq K h^{m}|u|_{H^{m+1}(\Omega)}, \quad\left\|v-\mathcal{I}_{v} v\right\|_{Z^{\prime}} \leq K h^{m}|v|_{H^{m+1}(\Omega)^{2}} .
$$

Proof. Let $\mathcal{I}_{u} u \in U_{h}$ be the interpolant of $u$. By the definition of $Z$-norm,

$$
\left\|\mathcal{I}_{u} u-u\right\|_{Z}^{2}=\int_{\Omega}\left(B^{*}\left(\mathcal{I}_{u} u-u\right)\right)^{2} d x+\sum_{\sigma \in \mathcal{E}_{v}^{0}}\left(h_{\sigma}\right)^{-1} \int_{\sigma}\left[\mathcal{I}_{u} u-u\right]^{2} d \sigma .
$$

The first term will be estimated by using the inverse inequality and the interpolation estimate (2.5)

$$
\int_{\Omega}\left(B^{*}\left(\mathcal{I}_{u} u-u\right)\right)^{2} d x \leq K h^{2 k}|u|_{H^{k+1}(\Omega)}^{2} .
$$

For the second term, we will use the trace inequality

$$
\begin{aligned}
\int_{\sigma}\left(\mathcal{I}_{u} u-u\right)^{2} d \sigma \leq & K\left(\left\|\mathcal{I}_{u} u-u\right\|_{L^{2}(\mathcal{R}(\tau))}\left\|\nabla\left(\mathcal{I}_{u} u-u\right)\right\|_{L^{2}(\mathcal{R}(\tau))}\right. \\
& \left.+h^{-1}\left\|\mathcal{I}_{u} u-u\right\|_{L^{2}(\mathcal{R}(\tau))}^{2}\right) .
\end{aligned}
$$

So,

$$
\int_{\sigma}\left[\mathcal{I}_{u} u-u\right]^{2} d \sigma \leq K h^{2 k+1}|u|_{H^{k+1}(\Omega)}^{2}
$$

and this implies

$$
\left\|\mathcal{I}_{u} u-u\right\|_{Z} \leq K h^{k}|u|_{H^{k+1}(\Omega)} .
$$

The estimate for $\left\|v-\mathcal{I}_{v} v\right\|_{Z^{\prime}}$ can be proved by a similar argument. 
4. Stability and convergence analysis. In this section, we will prove the stability and convergence of the scheme (3.3)-(3.4). We write $\langle u, v\rangle=\int_{\Omega} u v d x$ and $\|u\|=\langle u, u\rangle^{\frac{1}{2}}$. In order to obtain an optimal error estimate, we will assume the following.

\section{The inf-sup conditions:}

$$
\begin{array}{ll}
\inf _{\psi \in V_{h}} & \sup _{\phi \in U_{h}} \frac{B_{h}(\psi, \phi)}{\|\psi\|_{Z^{\prime}}\|\phi\|_{W}} \geq K, \\
\inf _{\phi \in U_{h}} & \sup _{\psi \in V_{h}} \frac{B_{h}^{*}(\phi, \psi)}{\|\phi\|_{Z}\|\psi\|_{W^{\prime}}} \geq K .
\end{array}
$$

For a general introduction to this topic; see Brezzi and Fortin [2].

Consequently, we have

$$
\begin{aligned}
\left\|B_{h} \psi\right\|_{W} & \geq K\|\psi\|_{Z^{\prime}}, \\
\left\|B_{h}^{*} \phi\right\|_{W^{\prime}} & \geq K\|\phi\|_{Z} .
\end{aligned}
$$

By the continuity conditions (3.12) and (3.13), we have

$$
\begin{aligned}
K_{1}\|\psi\|_{Z^{\prime}} & \leq\left\|B_{h} \psi\right\|_{W} \leq K_{2}\|\psi\|_{Z^{\prime}} \\
K_{1}\|\phi\|_{Z} & \leq\left\|B_{h}^{*} \phi\right\|_{W^{\prime}} \leq K_{2}\|\phi\|_{Z} .
\end{aligned}
$$

So, the discrete $H^{1}$ norm is equivalent to the discrete $L^{2}$ norm of the discrete derivative operator. Notice that, the above two inf-sup conditions (4.3)-(4.4) imply the existence of projection operators $\mathcal{P}_{v}$ and $\mathcal{P}_{u}$ such that

$$
\begin{aligned}
B_{h}\left(\mathcal{P}_{v} v-v, \phi\right)=0 & \forall \phi \in U_{h}, \\
B_{h}^{*}\left(\mathcal{P}_{u} u-u, \psi\right)=0 & \forall \psi \in V_{h} .
\end{aligned}
$$

Regarding the initial condition $u_{h}(0)$ and $v_{h}(0)$, we can obtain them by solving the following:

$$
\begin{aligned}
B_{h}\left(\mathcal{P}_{v} v_{0}-v_{0}, \phi\right)=0 & \forall \phi \in U_{h}, \\
B_{h}^{*}\left(\mathcal{P}_{u} u_{0}-u_{0}, \psi\right)=0 & \forall \psi \in V_{h} ;
\end{aligned}
$$

then set $u_{h}(0)=\mathcal{P}_{u} u_{0}$ and $v_{h}(0)=\mathcal{P}_{v} v_{0}$. However, in order to retain the accuracy of the approximation, the initial conditions can also be defined as $u_{h}(0)=\mathcal{I}_{u} u_{0}$ and $v_{h}(0)=\mathcal{I}_{v} v_{0}$, where $\mathcal{I}_{u}$ and $\mathcal{I}_{v}$ are some interpolation operators with the same order of accuracy and stability estimates $\left\|u_{h}(0)\right\| \leq K\left\|u_{0}\right\|$ and $\left\|v_{h}(0)\right\| \leq K\left\|v_{0}\right\|$.

One important property of the numerical approximation (3.3)-(3.4) is that energy is conserved, as is the case for the continuous problem (1.1)-(1.2). In particular, the method (3.3)-(3.4) is stable in the discrete $L^{2}$ norm. Moreover, the convergence in the $L^{2}$ norm is optimal. We state these results in the following theorem.

TheOrem 4.1. Let $u \in U$ and $v \in V$ be the solution to (1.1)-(1.2) and let $u_{h} \in U_{h}$ and $v_{h} \in V_{h}$ be the solution to the numerical scheme (3.3)-(3.4). Then, energy is conserved, namely

$$
\frac{d}{d t}\left(\left\|u_{h}\right\|^{2}+\left\|v_{h}\right\|^{2}\right)=0
$$


Moreover, for $0 \leq t \leq T$, we have

$$
\begin{aligned}
& \left\|\left(u-u_{h}\right)(t)\right\|+\left\|\left(v-v_{h}\right)(t)\right\| \\
& \leq K\left\{\inf _{\phi \in U_{h}}\|u-\phi\|_{W}+\inf _{\psi \in V_{h}}\|v-\psi\|_{W^{\prime}}\right. \\
& \left.\quad+\int_{0}^{t}\left(\inf _{\phi \in U_{h}}\left\|u_{t}-\phi\right\|_{W}+\inf _{\psi \in V_{h}}\left\|v_{t}-\psi\right\|_{W^{\prime}}\right) d s\right\} .
\end{aligned}
$$

Proof. Taking $\phi=u_{h}$ and $\psi=v_{h}$ in (3.3)-(3.4) yields

$$
\begin{aligned}
& a_{1}\left\langle\frac{d u_{h}}{d t}, u_{h}\right\rangle+B_{h}\left(v_{h}, u_{h}\right)=0, \\
& a_{2}\left\langle\frac{d v_{h}}{d t}, v_{h}\right\rangle-B_{h}^{*}\left(u_{h}, v_{h}\right)=0 .
\end{aligned}
$$

Adding the two equations and using (3.7), we obtain (4.11).

In the following, we will prove (4.12). Let $\mathcal{I}_{u} u$ and $\mathcal{I}_{v} v$ be arbitrary elements in $U_{h}$ and $V_{h}$, respectively. First, we have

$$
\begin{array}{cc}
a_{1}\left\langle\frac{d\left(u-u_{h}\right)}{d t}, \phi\right\rangle+B_{h}\left(v-v_{h}, \phi\right)=0 & \forall \phi \in U_{h}, \\
a_{2}\left\langle\frac{d\left(v-v_{h}\right)}{d t}, \psi\right\rangle-B_{h}^{*}\left(u-u_{h}, \psi\right)=0 & \forall \psi \in V_{h} .
\end{array}
$$

By the definitions of the projection operators $\mathcal{P}_{u}$ and $\mathcal{P}_{v}$, we obtain

$$
\begin{array}{cc}
a_{1}\left\langle\frac{d\left(u-u_{h}\right)}{d t}, \phi\right\rangle+B_{h}\left(\mathcal{P}_{v} v-v_{h}, \phi\right)=0 & \forall \phi \in U_{h}, \\
a_{2}\left\langle\frac{d\left(v-v_{h}\right)}{d t}, \psi\right\rangle-B_{h}^{*}\left(\mathcal{P}_{u} u-u_{h}, \psi\right)=0 & \forall \psi \in V_{h} .
\end{array}
$$

Let $Q_{u}^{2}: U_{h} \rightarrow \operatorname{ker}\left(B_{h}^{*}\right)^{\perp}$ and $Q_{v}^{2}: V_{h} \rightarrow \operatorname{ker}\left(B_{h}\right)^{\perp}$ be the projection operators. Taking $\phi=Q_{u}^{2}\left(\mathcal{P}_{u} u-u_{h}\right)$ and $\psi=Q_{v}^{2}\left(\mathcal{P}_{v} v-v_{h}\right)$, we have, by adding the two equations,

$$
a_{1}\left\langle\frac{d\left(u-u_{h}\right)}{d t}, Q_{u}^{2}\left(\mathcal{P}_{u} u-u_{h}\right)\right\rangle+a_{2}\left\langle\frac{d\left(v-v_{h}\right)}{d t}, Q_{v}^{2}\left(\mathcal{P}_{v} v-v_{h}\right)\right\rangle=0,
$$

which implies

$$
\begin{aligned}
& \frac{d}{d t}\left(a_{1}\left\|Q_{u}^{2}\left(\mathcal{P}_{u} u-u_{h}\right)\right\|^{2}+a_{2}\left\|Q_{v}^{2}\left(\mathcal{P}_{v} v-v_{h}\right)\right\|^{2}\right) \\
& \quad=a_{1}\left\langle\frac{d\left(\mathcal{P}_{u} u-u\right)}{d t}, Q_{u}^{2}\left(\mathcal{P}_{u} u-u_{h}\right)\right\rangle+a_{2}\left\langle\frac{d\left(\mathcal{P}_{v} v-v\right)}{d t}, Q_{v}^{2}\left(\mathcal{P}_{v} v-v_{h}\right)\right\rangle
\end{aligned}
$$

This can be rewritten as

$$
\begin{aligned}
\frac{d}{d t}( & \left.a_{1}\left\|Q_{u}^{2}\left(\mathcal{P}_{u} u-u_{h}\right)\right\|^{2}+a_{2}\left\|Q_{v}^{2}\left(\mathcal{P}_{v} v-v_{h}\right)\right\|^{2}\right) \\
= & a_{1}\left\langle\frac{d\left(\mathcal{P}_{u} u-\mathcal{I}_{u} u\right)}{d t}, Q_{u}^{2}\left(\mathcal{P}_{u} u-u_{h}\right)\right\rangle+a_{2}\left\langle\frac{d\left(\mathcal{P}_{v} v-\mathcal{I}_{v} v\right)}{d t}, Q_{v}^{2}\left(\mathcal{P}_{v} v-v_{h}\right)\right\rangle \\
& +a_{1}\left\langle\frac{d\left(\mathcal{I}_{u} u-u\right)}{d t}, Q_{u}^{2}\left(\mathcal{P}_{u} u-u_{h}\right)\right\rangle+a_{2}\left\langle\frac{d\left(\mathcal{I}_{v} v-v\right)}{d t}, Q_{v}^{2}\left(\mathcal{P}_{v} v-v_{h}\right)\right\rangle .
\end{aligned}
$$


Consequently,

$$
\begin{aligned}
& \left\|a_{1} Q_{u}^{2}\left(\mathcal{P}_{u} u-u_{h}\right)\right\|+\left\|a_{2} Q_{v}^{2}\left(\mathcal{P}_{v} v-v_{h}\right)\right\| \\
& \leq K \int_{0}^{t}\left\{\left\|Q_{u}^{2}\left(\mathcal{P}_{u} u_{t}-\mathcal{I}_{u} u_{t}\right)\right\|+\left\|Q_{v}^{2}\left(\mathcal{P}_{v} v_{t}-\mathcal{P}_{v} v_{t}\right)\right\|\right\} d s \\
& \quad+K \int_{0}^{t}\left\{\left\|\mathcal{I}_{u} u_{t}-u_{t}\right\|+\left\|\mathcal{I}_{v} v_{t}-v_{t}\right\|\right\} d s
\end{aligned}
$$

Using the triangle inequality, we finally have

$$
\begin{aligned}
&\left\|a_{1} Q_{u}^{2}\left(\mathcal{I}_{u} u-u_{h}\right)\right\|+\left\|a_{2} Q_{v}^{2}\left(\mathcal{I}_{v} v-v_{h}\right)\right\| \\
& \leq K\left(\left\|Q_{u}^{2}\left(\mathcal{P}_{u} u-\mathcal{I}_{u} u\right)\right\|+\left\|Q_{v}^{2}\left(\mathcal{P}_{v} v-\mathcal{I}_{v} v\right)\right\|\right) \\
&+K \int_{0}^{t}\left\{\left\|Q_{u}^{2}\left(\mathcal{P}_{u} u_{t}-\mathcal{I}_{u} u_{t}\right)\right\|+\left\|Q_{v}^{2}\left(\mathcal{P}_{v} v_{t}-\mathcal{P}_{v} v_{t}\right)\right\|\right\} d s \\
&+K \int_{0}^{t}\left\{\left\|\mathcal{I}_{u} u_{t}-u_{t}\right\|+\left\|\mathcal{I}_{v} v_{t}-v_{t}\right\|\right\} d s .
\end{aligned}
$$

It suffices to estimate the norms $\left\|Q_{v}^{2}\left(\mathcal{P}_{v} v-\mathcal{I}_{v} v\right)\right\|$ and $\left\|Q_{u}^{2}\left(\mathcal{P}_{u} u-\mathcal{I}_{u} u\right)\right\|$. In particular, we will prove $\left\|Q_{v}^{2}\left(\mathcal{P}_{v} v-\mathcal{I}_{v} v\right)\right\| \leq K\left\|v-\mathcal{I}_{v} v\right\|_{W^{\prime}}$ and $\left\|Q_{u}^{2}\left(\mathcal{P}_{u} u-\mathcal{I}_{u} u\right)\right\| \leq K\left\|u-\mathcal{I}_{u} u\right\|_{W}$.

We consider the following variational problem: Given $u_{2} \in \operatorname{ker}\left(B_{h}^{*}\right)^{\perp}$, find $\tilde{\psi} \in V_{h}$ such that

$$
B_{h}(\tilde{\psi}, \phi)=\left\langle u_{2}, \phi\right\rangle \quad \forall \phi \in U_{h}
$$

The existence of $\tilde{\psi}$ is ensured by the fact that $B_{h}: V_{h} \rightarrow \operatorname{ker}\left(B_{h}^{*}\right)^{\perp}$ is surjective.

Taking the supremum in $\phi$ and using (4.3), we derive the following estimate:

$$
\|\tilde{\psi}\|_{Z^{\prime}} \leq K\left\|u_{2}\right\|
$$

Now, we have

$$
\begin{aligned}
\left\|Q_{u}^{2}\left(\mathcal{P}_{u} u-\mathcal{I}_{u} u\right)\right\|^{2} & =B_{h}\left(\tilde{\psi}, Q_{u}^{2}\left(\mathcal{P}_{u} u-\mathcal{I}_{u} u\right)\right) \\
& =B_{h}^{*}\left(Q_{u}^{2}\left(\mathcal{P}_{u} u-\mathcal{I}_{u} u\right), \tilde{\psi}\right) \\
& =B_{h}^{*}\left(\mathcal{P}_{u} u-\mathcal{I}_{u} u, \tilde{\psi}\right) \\
& =B_{h}^{*}\left(u-\mathcal{I}_{u} u, \tilde{\psi}\right) \\
& \leq\|\psi\|_{Z^{\prime}}\left\|u-\mathcal{I}_{u} u\right\|_{W} \\
& \leq K\left\|Q_{u}^{2}\left(\mathcal{P}_{u} u-\mathcal{I}_{u} u\right)\right\|\left\|u-\mathcal{I}_{u} u\right\|_{W} .
\end{aligned}
$$

Hence, we have

$$
\left\|Q_{u}^{2}\left(\mathcal{P}_{u} u-\mathcal{I}_{u} u\right)\right\| \leq\left\|u-\mathcal{I}_{u} u\right\|_{W} .
$$

Replacing $u$ by $u_{t}$, we have

$$
\left\|Q_{u}^{2}\left(\mathcal{P}_{u} u_{t}-\mathcal{I}_{u} u_{t}\right)\right\| \leq\left\|u_{t}-\mathcal{I}_{u} u_{t}\right\|_{W} .
$$

Similarly, we consider the problem: Given $v_{2} \in \operatorname{ker}\left(B_{h}\right)^{\perp}$, find $\tilde{\phi} \in U_{h}$ such that

$$
B_{h}^{*}(\tilde{\phi}, \psi)=\left\langle v_{2}, \psi\right\rangle \quad \forall \psi \in V_{h}
$$


with the estimate

$$
\|\tilde{\phi}\|_{Z} \leq K\left\|v_{2}\right\|
$$

Hence, we have

$$
\left\|Q_{v}^{2}\left(\mathcal{P}_{v} v-\mathcal{I}_{v} v\right)\right\| \leq\left\|v-\mathcal{I}_{v} v\right\|_{W^{\prime}} .
$$

Replacing $v$ by $v_{t}$, we have

$$
\left\|Q_{v}^{2}\left(\mathcal{P}_{v} v_{t}-\mathcal{I}_{v} v_{t}\right)\right\| \leq\left\|v_{t}-\mathcal{I}_{v} v_{t}\right\|_{W^{\prime}} .
$$

This finishes the proof of estimates of the components of the errors in $\operatorname{ker}\left(B_{h}^{*}\right)^{\perp}$ and $\operatorname{ker}\left(B_{h}\right)^{\perp}$. In the following, we will estimate the components of errors in $\operatorname{ker}\left(B_{h}^{*}\right)$ and $\operatorname{ker}\left(B_{h}\right)$.

Define $Q_{u}^{1}: U_{h} \rightarrow \operatorname{ker}\left(B_{h}^{*}\right)$ and $Q_{v}^{1}: V_{h} \rightarrow \operatorname{ker}\left(B_{h}\right)$ as the orthogonal projection operators. Taking $\phi=Q_{u}^{1}\left(\mathcal{I}_{u} u-u_{h}\right)$ and $\psi=Q_{v}^{1}\left(\mathcal{I}_{v} v-v_{h}\right)$, we have

$$
a_{1}\left\langle\frac{d\left(u-u_{h}\right)}{d t}, Q_{u}^{1}\left(\mathcal{I}_{u} u-u_{h}\right)\right\rangle+a_{2}\left\langle\frac{d\left(v-v_{h}\right)}{d t}, Q_{v}^{1}\left(\mathcal{I}_{v} v-v_{h}\right)\right\rangle=0 .
$$

Hence, we obtain

$$
\left\|a_{1} Q_{u}^{1}\left(\mathcal{I}_{u} u-u_{h}\right)\right\|+\left\|a_{2} Q_{v}^{1}\left(\mathcal{I}_{v} v-v_{h}\right)\right\| \leq \int_{0}^{t}\left\{\left\|\mathcal{I}_{u} u_{t}-u_{t}\right\|+\left\|\mathcal{I}_{v} v_{t}-v_{t}\right\|\right\} d s .
$$

Combining all results,

$$
\begin{aligned}
\left\|\mathcal{I}_{u} u-u_{h}\right\|_{u}+\left\|\mathcal{I}_{v} v-v_{h}\right\|_{v} \leq K\{ & \left\|Q_{u}^{1}\left(\mathcal{I}_{u} u-u_{h}\right)\right\|_{u}+\left\|Q_{u}^{2}\left(\mathcal{I}_{u} u-u_{h}\right)\right\|_{u} \\
& \left.+\left\|Q_{v}^{1}\left(\mathcal{I}_{v} v-v_{h}\right)\right\|_{v}+\left\|Q_{v}^{2}\left(\mathcal{I}_{v} v-v_{h}\right)\right\|_{v}\right\} .
\end{aligned}
$$

The proof is complete by noticing that

$$
\begin{aligned}
\left\|u-u_{h}\right\| & \leq\left\|u-\mathcal{I}_{u} u\right\|+\left\|\mathcal{I}_{u} u-u_{h}\right\|, \\
\left\|v-v_{h}\right\| & \leq\left\|v-\mathcal{I}_{v} v\right\|+\left\|\mathcal{I}_{v} v-v_{h}\right\| .
\end{aligned}
$$

Now, we will state the convergence theorems. The following is the convergence in $L^{2}$ norm. We see that the numerical scheme is $O\left(h^{k+1}\right)$ when the FE spaces $U_{h}$ and $V_{h}$ contain polynomials of degree $k$.

Corollary 4.2. Let $(u, v) \in W^{1,1}\left(0, T ; W^{k+1, \infty}(\Omega)\right)^{3}$ be the exact solution to the wave propagation problem (1.1)-(1.2) and let $\left(u_{h}, v_{h}\right)$ be the solution to (3.3)-(3.4). Then

$$
\left\|u-u_{h}\right\|+\left\|v-v_{h}\right\| \leq K h^{k+1}\left(\|u\|_{W^{1,1}\left(0, T ; W^{k+1, \infty}(\Omega)\right)}+\|v\|_{W^{1,1}\left(0, T ; W^{k+1, \infty}(\Omega)\right)^{2}}\right) .
$$

Theorem 4.1 and Corollary 4.2 state that the numerical scheme (3.3)-(3.4) is stable and convergent, with optimal rate, with respect to the discrete $L^{2}$-norms. The $L^{2}$ stability can be satisfied by a very large class of spaces $U_{h}$ and $V_{h}$. However, with $L^{2}$ stability only, it is not sufficient to deduce the weak convergence to the true solution; 
see Joly [11]. As a result, the numerical solution may behave badly and the optimal rate of convergence is not achieved. This fact can be seen by some numerical examples in the following sections. The extra conditions needed are the inf-sup conditions (4.1)(4.2), as many mixed FE methods require some compatibility conditions between the two spaces $U_{h}$ and $V_{h}$. These conditions yield a stability in the discrete $H^{1}$ norm and we state this result in the following theorem.

THEOREM 4.3. Let $u_{h} \in U_{h}$ and $v_{h} \in V_{h}$ be the solution to the numerical scheme (3.3)-(3.4). Then

$$
\left\|u_{h}\right\|_{Z}+\left\|v_{h}\right\|_{Z^{\prime}} \leq K\left\{\left\|\frac{d u_{0}}{d t}\right\|+\left\|\frac{d v_{0}}{d t}\right\|+\int_{0}^{t}\left(\left\|\frac{d F_{1}}{d t}\right\|+\left\|\frac{d F_{2}}{d t}\right\|\right) d s\right\} .
$$

Proof. Taking $t$-derivative in (3.3)-(3.4), we have

$$
\begin{array}{ll}
a_{1}\left\langle\frac{d^{2} u_{h}}{d t^{2}}, \phi\right\rangle+B_{h}\left(\frac{d v_{h}}{d t}, \phi\right)=\left\langle\frac{d F_{1}}{d t}, \phi\right\rangle \quad \forall \phi \in U_{h}, \\
a_{2}\left\langle\frac{d^{2} v_{h}}{d t^{2}}, \psi\right\rangle-B_{h}^{*}\left(\frac{d u_{h}}{d t}, \psi\right)=\left\langle\frac{d F_{2}}{d t}, \psi\right\rangle \quad \forall \psi \in V_{h} .
\end{array}
$$

Taking $\phi=\frac{d u_{h}}{d t}$ and $\psi=\frac{d v_{h}}{d t}$ and adding the two equations, we obtain

$$
\frac{1}{2} \frac{d}{d t}\left(a_{1}\left\|\frac{d u_{h}}{d t}\right\|^{2}+a_{2}\left\|\frac{d v_{h}}{d t}\right\|^{2}\right)=\left\langle\frac{d F_{1}}{d t}, \frac{d u_{h}}{d t}\right\rangle+\left\langle\frac{d F_{2}}{d t}, \frac{d v_{h}}{d t}\right\rangle,
$$

and consequently

$$
\left\|a_{1} \frac{d u_{h}}{d t}\right\|+\left\|a_{2} \frac{d v_{h}}{d t}\right\| \leq K\left\{\left\|\frac{d u_{0}}{d t}\right\|+\left\|\frac{d v_{0}}{d t}\right\|+\int_{0}^{t}\left(\left\|\frac{d F_{1}}{d t}\right\|+\left\|\frac{d F_{2}}{d t}\right\|\right) d s\right\} .
$$

By using (4.3),

$$
\left\|v_{h}\right\|_{Z^{\prime}} \leq K\left\|B_{h} v_{h}\right\|_{W}=K \sup _{\phi \in U_{h}} \frac{\left|B_{h}\left(v_{h}, \phi\right)\right|}{\|\phi\|_{W}} \leq K \sup _{\phi \in U_{h}} \frac{\left|\left\langle\frac{d u_{h}}{d t}, \phi\right\rangle\right|}{\|\phi\|_{W}}=K\left\|\frac{d u_{h}}{d t}\right\| .
$$

Similarly, we have

$$
\left\|u_{h}\right\|_{Z} \leq K\left\|\frac{d v_{h}}{d t}\right\| .
$$

This completes the proof.

Before we state the convergence theorem, we will state a $L^{2}$ convergence result which is very similar to Theorem 4.1-that all functions are replaced by their time derivative. It can be proved in exactly the same way as the proof of Theorem 4.1.

LEMma 4.4. Let $u \in U$ and $v \in V$ be the solution to (1.1)-(1.2) and let $u_{h} \in U_{h}$ and $v_{h} \in V_{h}$ be the solution to the numerical scheme (3.3)-(3.4). Then for $0 \leq t \leq T$, we have

$$
\begin{aligned}
\left\|\frac{d}{d t}\left(u-u_{h}\right)(t)\right\|+\left\|\frac{d}{d t}\left(v-v_{h}\right)(t)\right\| \leq & K\left\{\inf _{\phi \in U_{h}}\left\|u_{t}-\phi\right\|_{W}+\inf _{\psi \in V_{h}}\left\|v_{t}-\psi\right\|_{W^{\prime}}\right. \\
& \left.+\int_{0}^{t}\left(\inf _{\phi \in U_{h}}\left\|u_{t t}-\phi\right\|_{W}+\inf _{\psi \in V_{h}}\left\|v_{t t}-\psi\right\|_{W^{\prime}}\right) d s\right\} .
\end{aligned}
$$


The following theorem states the convergence of the method (3.3)-(3.4) in the discrete $H^{1}$ norm. It can be seen that the $H^{1}$ error is optimal with respect to the norms and the FE spaces.

THEOREM 4.5. Let $u \in U$ and $v \in V$ be the solution to (1.1)-(1.2) and let $u_{h} \in U_{h}$ and $v_{h} \in V_{h}$ be the solution to the numerical scheme (3.3)-(3.4). Then for $0 \leq t \leq T$, we have

$$
\begin{aligned}
& \left\|v-v_{h}\right\|_{Z^{\prime}} \leq K\left(\left\|\frac{d}{d t}\left(u-u_{h}\right)\right\|+\inf _{\psi \in V_{h}}\|\psi-v\|_{Z^{\prime}}\right), \\
& \left\|u-u_{h}\right\|_{Z} \leq K\left(\left\|\frac{d}{d t}\left(v-v_{h}\right)\right\|+\inf _{\phi \in U_{h}}\|\phi-u\|_{Z}\right) .
\end{aligned}
$$

Proof. By the inf-sup condition (4.3), we obtain

$$
\left\|\mathcal{P}_{v} v-v_{h}\right\|_{Z^{\prime}} \leq K\left\|B_{h}\left(\mathcal{P}_{v} v-v_{h}\right)\right\|_{W}=K \sup _{\phi \in U_{h}} \frac{B_{h}\left(\mathcal{P}_{v} v-v_{h}, \phi\right)}{\|\phi\|_{W}} .
$$

Recalling (4.15), we have

$$
\left\langle\frac{d\left(u-u_{h}\right)}{d t}, \phi\right\rangle+B_{h}\left(\mathcal{P}_{v} v-v_{h}, \phi\right)=0 \quad \forall \phi \in U_{h}
$$

and consequently,

$$
\left\|\mathcal{P}_{v} v-v_{h}\right\|_{Z^{\prime}} \leq K\left\|\frac{d}{d t}\left(u-u_{h}\right)\right\|
$$

Let $\mathcal{I}_{v} v \in V_{h}$ be an arbitrary element of the FE space $V_{h}$, using the triangle inequality

$$
\left\|v-v_{h}\right\|_{Z^{\prime}} \leq\left\|v-\mathcal{I}_{v} v\right\|_{Z^{\prime}}+\left\|\mathcal{I}_{v} v-\mathcal{P}_{v} v\right\|_{Z^{\prime}}+\left\|\mathcal{P}_{v} v-v_{h}\right\|_{Z^{\prime}}
$$

Following the proof of Theorem 4.1, we have

$$
\left\|\mathcal{I}_{v} v-\mathcal{P}_{v} v\right\|_{Z^{\prime}} \leq K\left\|\mathcal{I}_{v} v-v\right\|_{Z^{\prime}}
$$

Hence, we obtain

$$
\left\|v-v_{h}\right\|_{Z^{\prime}} \leq K\left(\left\|\frac{d}{d t}\left(u-u_{h}\right)\right\|+\left\|\mathcal{I}_{v} v-v\right\|_{Z^{\prime}}\right) .
$$

Since $\mathcal{I}_{v} v$ is arbitrary,

$$
\left\|v-v_{h}\right\|_{Z^{\prime}} \leq K\left(\left\|\frac{d}{d t}\left(u-u_{h}\right)\right\|+\inf _{\psi \in V_{h}}\|\psi-v\|_{Z^{\prime}}\right) .
$$

So, (4.29) is proved. The estimte (4.30) can be proved in a similar fashion.

Now, we state and prove the convergence in the discrete $H^{1}$ norm. We see that the numerical scheme is $O\left(h^{k}\right)$ in the discrete $H^{1}$ norm when the FE spaces $U_{h}$ and $V_{h}$ contain polynomials of degree $k$.

Corollary 4.6. Assume $k \geq 0$ is the largest integer such that $U_{h}$ and $V_{h}$ contain polynomials of degree $k$. Let $(u, v) \in W^{1, p}\left(0, T ; H^{k+1}(\Omega)\right)^{3} \cap W^{2, p}\left(0, T ; W^{k, \infty}(\Omega)\right)^{3}$, 
for $p>1$, be the exact solution to the wave propagation problem (1.1)-(1.2), and let $\left(u_{h}, v_{h}\right)$ be the solution to the numerical scheme (3.3)-(3.4). Then

$$
\begin{aligned}
\left\|u-u_{h}\right\|_{Z}+\left\|v-v_{h}\right\|_{Z^{\prime}} \leq & K h^{k}\left(\|(u, v)\|_{W^{1, p}\left(0, T ; H^{k+1}(\Omega)\right)^{3}}\right. \\
& +\|(u, v)\|_{W^{2, p}\left(0, T ; W^{k, \infty}(\Omega)\right)^{3}} .
\end{aligned}
$$

Next, we prove a superconvergence result for some component of the derivative of $u_{h}$. We state this result as the following theorem.

THEOREM 4.7. Let $u$ be the exact solution to the wave propagation problem (1.1)(1.2) and let $u_{h}$ be the solution to the numerical method (3.3)-(3.4). Then

$$
\begin{aligned}
& \left\|L^{\perp} B^{*}\left(u-u_{h}\right)\right\|_{L^{2}(\Omega)} \\
& \quad \leq K h^{k+1}\left\{\|(u, v)\|_{W^{2,1}\left(0, T ; W^{k+1, \infty}(\Omega)\right)^{3}}+\|u\|_{W^{1,1}\left(0, T ; H^{k+2}(\Omega)\right)}\right\} .
\end{aligned}
$$

Proof. To prove this, we observe that for all $\psi \in V_{h}$

$$
a_{2} \int_{\Omega} \frac{\partial}{\partial t}\left(v-v_{h}\right) \cdot \psi d x-B_{h}^{*}\left(u-u_{h}, \psi\right)=0 .
$$

Let $\pi_{h} u$ be a function such that $\left.\pi_{h} u\right|_{\tau}$ is a $(k+1)$ th degree polynomial interpolant of $\left.u\right|_{\tau}$. Then we have

$$
B_{h}^{*}\left(u_{h}-\pi_{h} u, \psi\right)=B_{h}^{*}\left(u-\pi_{h} u, \psi\right)-a_{2} \int_{\Omega} \frac{\partial}{\partial t}\left(v-v_{h}\right) \cdot \psi d x .
$$

Recalling the definition of $B_{h}^{*}$, we have

$$
B_{h}^{*}\left(u_{h}-\pi_{h} u, \psi\right)=\int_{\Omega} B^{*}\left(u_{h}-\pi_{h} u\right) \psi d x+\sum_{\sigma \in \mathcal{E}_{u}} \int_{\sigma}\left[u_{h}-\pi_{h} u\right] L \psi d \sigma .
$$

Now, we choose $\psi \in V_{h}$ such that $L \psi=0$ and $L^{\perp} \psi=L^{\perp} B^{*}\left(u_{h}-\pi_{h} u\right)$. This is equivalent to sets (VD2) and (VD3) to being zero. Therefore,

$$
\begin{aligned}
B_{h}^{*}\left(u_{h}-\pi_{h} u, \psi\right) & =\int_{\Omega}\left(L^{\perp} B^{*}\left(u_{h}-\pi_{h} u\right)\right)^{2} d x, \\
\|\psi\|_{L^{2}(\Omega)} & =\left\|L^{\perp} B^{*}\left(u_{h}-\pi_{h} u\right)\right\|_{L^{2}(\Omega)} .
\end{aligned}
$$

Consequently, we have

$$
\left\|L^{\perp} B^{*}\left(u_{h}-\pi_{h} u\right)\right\|_{L^{2}(\Omega)}^{2}=B_{h}^{*}\left(u-\pi_{h} u, \psi\right)-a_{2} \int_{\Omega} \frac{\partial}{\partial t}\left(v-v_{h}\right) \cdot \psi d x .
$$

Now we will estimate the right-hand side of (4.33). By Lemma 4.4 and interpolation error estimates (3.16), the second term on the right-hand side of (4.33) can be estimated by

$$
\left\|\frac{\partial}{\partial t}\left(v-v_{h}\right)\right\|_{L^{2}(\Omega)} \leq K h^{k+1}\left(\|u\|_{W^{2,1}\left(0, T ; W^{k+1, \infty}(\Omega)\right)}+\|v\|_{W^{2,1}\left(0, T ; W^{k+1, \infty}(\Omega)\right)^{2}}\right) .
$$

Using inverse type inequalities, we have

$$
\left\|B^{*}\left(u-\pi_{h} u\right)\right\|_{L^{2}(\Omega)} \leq K h^{k+1}|u|_{H^{k+2}(\Omega)}, \quad\|\psi \cdot l\|_{L^{2}(\sigma)} \leq K h^{-\frac{1}{2}}\|\psi\|_{L^{2}\left(\tau^{\prime}\right)},
$$


and by the trace inequality, we obtain

$$
\left\|u-\pi_{h} u\right\|_{L^{2}(\sigma)} \leq K h^{k+\frac{3}{2}}|u|_{H^{k+2}(\Omega)} .
$$

Then the first term on the right-hand side of (4.33) can be estimated by

$$
\begin{aligned}
B_{h}^{*}\left(u-\pi_{h} u, \psi\right) \leq & \left\|B^{*}\left(u-\pi_{h} u\right)\right\|_{L^{2}(\Omega)}\|\psi\|_{L^{2}(\Omega)} \\
& +\sum_{\sigma \in \mathcal{E}_{u}}\left\|u-\pi_{h} u\right\|_{L^{2}(\sigma)}\|L \psi\|_{L^{2}(\sigma)} \\
\leq & K h^{k+1}|u|_{H^{k+2}(\Omega)}\|\psi\|_{L^{2}(\Omega)} .
\end{aligned}
$$

Combining the result, we prove the theorem.

Now, we will discuss the condition (1.4). In the following theorem, we show that the numerical solution $v_{h}$ satisfies (1.4) in a weak sense. Let $S_{h}$ be the space of standard $H^{1}$-conforming FE space of degree $k+1$, namely $p \in S_{h}$ if $\left.p\right|_{\tau} \in P^{k+1}(\tau)$ and $p$ is continuous across each $\sigma \in \mathcal{E}$.

THEOREM 4.8. Let $v$ be the exact solution of the wave propagation problem (1.1)(1.2) and let $v_{h}$ be the numerical solution to the numerical scheme (3.3)-(3.4). Then

$$
\int_{\Omega} \frac{\partial\left(v-v_{h}\right)}{\partial t} \psi d x=0
$$

if and only if $\psi=B^{\perp} p$ for $p \in S_{h}$.

Proof. If $\psi=\nabla p$ for $p \in S_{h}$, then $\psi \in V_{h}$. Using (4.16), we have proved (4.34). Assume (4.34) holds. Then, using (4.16), we have $B_{h}^{*}\left(\mathcal{P}_{u} u-u_{h}, \psi\right)=0$. By (4.3), we have $\|\psi\|_{Z^{\prime}}=0$. By the definition of $Z^{\prime}$-norm, we have $B \psi=0$ and $\left.[L \psi]\right|_{\sigma}=0$ for all $\sigma \in \mathcal{E}_{u}$. Using $B \psi=0$, we have $\psi=B^{\perp} p$ for some $p$. Since $\left.\psi\right|_{\tau} \in P^{k}(\tau)^{2}$, we have $\left.p\right|_{\tau} \in P^{k+1}(\tau)$. Notice that $L \psi$ is continuous on each edge in $\mathcal{E}_{v}^{0}$. Using this and $\left.[L \psi]\right|_{\sigma}=0$ for all $\sigma \in \mathcal{E}_{u}$, we have that $p$ is continuous across each edge in $\mathcal{E}_{u} \cup \mathcal{E}_{v}^{0}$. So, the proof is complete.

5. Verification of inf-sup conditions. Now, we are in a position to prove that the choice of $U_{h}$ and $V_{h}$ above satisfies the inf-sup condition (4.1)-(4.2).

THEOREM 5.1. There is a uniform constant $K>0$ such that

$$
\inf _{\psi \in V_{h}} \sup _{u \in U_{h}} \frac{B_{h}^{*}(u, \psi)}{\|u\|_{W}\|\psi\|_{Z^{\prime}}} \geq K .
$$

Proof. Let $\psi \in V_{h}$. It suffices to find $u \in U_{h}$ such that

$$
B_{h}^{*}(u, \psi) \geq K\|\psi\|_{Z^{\prime}}^{2} \quad \text { and } \quad\|u\| \leq K\|\psi\|_{Z^{\prime}} .
$$

Recalling the definition of $B_{h}^{*}$, we have

$$
B_{h}^{*}(u, \psi)=\int_{\Omega} u(B \psi) d x+\sum_{\sigma \in \mathcal{E}_{u}} \int_{\sigma}[L \psi] u d \sigma .
$$

First, we will define $u_{1} \in U_{h}$ such that

$$
\int_{\Omega} u_{1}(B \psi) d x \geq K \int_{\Omega}(B \psi)^{2} d x \quad \text { and } \quad \int_{\Omega}\left(u_{1}\right)^{2} d x \leq \int_{\Omega}(B \psi)^{2} d x .
$$


Let $\tau \in \mathcal{T}$. We define the function $u_{1}$ such that $\left.u_{1}\right|_{\tau}=\lambda_{\tau, 2} \lambda_{\tau, 3} B \psi$. Notice that $\left.B \psi\right|_{\tau} \in P^{k-1}(\tau)$ and $\left.u_{1}\right|_{\tau}$ is zero on the two edges of $\tau$ that belong to $\mathcal{E}_{u}$, so $\left.u_{1}\right|_{\tau} \in$ $R^{k}(\tau)$. Since $\lambda_{\tau, 2} \lambda_{\tau, 3} \leq 1$, the second equation in (5.3) holds. Notice that the quantity $\int_{\tau} q_{k-1}^{2} \lambda_{\tau, 2} \lambda_{\tau, 3} d x$ defines a norm for $q_{k-1}$ in the space $P^{k-1}(\tau)$. Since norms in finite dimensional spaces are equivalent, we have

$$
\int_{\tau} \lambda_{\tau, 2} \lambda_{\tau, 3}(B \psi)^{2} d x \geq K \int_{\tau}(B \psi)^{2} d x
$$

Summing up this equation for all $\tau \in \mathcal{T}$ proves the first equation in (5.3).

Next, we will define $u_{2} \in U_{h}$ such that

$$
\begin{aligned}
\sum_{\sigma \in \mathcal{E}_{u}} \int_{\sigma}[L \psi] u_{2} d \sigma & =\sum_{\sigma \in \mathcal{E}_{u}}\left(h_{\sigma}\right)^{-1} \int_{\sigma}[L \psi]^{2} d \sigma, \\
\int_{\Omega}\left(u_{2}\right)^{2} d x & \leq K \sum_{\sigma \in \mathcal{E}_{u}}\left(h_{\sigma}\right)^{-1} \int_{\sigma}[L \psi]^{2} d \sigma .
\end{aligned}
$$

To do so, we define $u_{2}$ so that

1. $u_{2}=\left(h_{\sigma}\right)^{-1}[L \psi]$ at the $k+1$ Gaussian points of $\sigma$ for all $\sigma \in \mathcal{E}_{u}$, and

2. $\int_{\tau} u_{2} q_{k-1} d x=0$ for all $q_{k-1} \in P^{k-1}(\tau)$ and $\tau \in \mathcal{T}$.

Then, clearly, (5.4) is satisfied. We will define a subspace $U_{h}^{0}$ of $U_{h}$ by

$$
U_{h}^{0}=\left\{\phi \in U_{h} \mid \int_{\tau} \phi q_{k-1} d x=0, \forall q_{k-1} \in P^{k-1}(\tau), \forall \tau \in \mathcal{T}\right\} .
$$

Then the following quantity

$$
\sum_{\sigma \in \mathcal{E}_{u}} h_{\sigma}^{2} \sum_{j=1}^{k+1} \phi\left(\alpha_{j}\right)^{2}
$$

defines a norm for $U_{h}^{0}$. Since norms in finite dimensional spaces are equivalent, we have

$$
\int_{\tau} u_{2}^{2} d x \leq K \sum_{\sigma \in \mathcal{E}_{u}} h_{\sigma}^{2} \sum_{j=1}^{k+1} u_{2}\left(\alpha_{j}\right)^{2} .
$$

By the definition of $u_{2}$,

$$
\int_{\tau} u_{2}^{2} d x \leq K \sum_{\sigma \in \mathcal{E}_{u}} \sum_{j=1}^{k+1}\left[L \psi\left(\alpha_{j}\right)\right]^{2} .
$$

Since $[L \psi]$ is a polynomial of degree $k$, we have

$$
\sum_{j=1}^{k+1}\left[L \psi\left(\alpha_{j}\right)\right]^{2} \leq K\left(h_{\sigma}\right)^{-1} \int_{\sigma}[L \psi]^{2} d x,
$$

which follows from norm equivalence in finite dimensional spaces. So, (5.5) is proved.

To prove (5.2), we take $u=u_{1}+u_{2}$. Notice that by the definitions of $u_{1}$ and $u_{2}$, we have

$$
\int_{\tau} u_{2}(B \psi) d x=0 \quad \text { and } \quad \int_{\sigma}[L \psi] u_{1} d \sigma=0
$$


Using this together with (5.3), (5.4), and (5.5), we have proved (5.2).

THEOREM 5.2. There is a uniform constant $K>0$ such that

$$
\inf _{\phi \in U_{h}} \sup _{v \in V_{h}} \frac{B_{h}(v, \phi)}{\|v\|_{W^{\prime}}\|\phi\|_{Z}} \geq K
$$

Proof. Let $\phi \in U_{h}$. As in the proof of the previous theorem, we find $v \in V_{h}$ such that

$$
B_{h}(v, \phi) \geq K\|\phi\|_{Z}^{2} \quad \text { and } \quad\|v\| \leq K\|\phi\|_{Z}
$$

Recalling the definition of $B_{h}$, we have

$$
B_{h}(v, \phi)=\int_{\Omega}\left(B^{*} \phi\right) v d x-\sum_{\sigma \in \mathcal{E}_{v}^{0}} \int_{\sigma}(L v)[\phi] d \sigma .
$$

We define $v_{1} \in V_{h}$ such that

$$
\int_{\Omega}\left(B^{*} \phi\right) v_{1} d x \geq K_{1} \int_{\Omega}\left|B^{*} \phi\right|^{2} d x \quad \text { and } \quad \int_{\Omega}\left|v_{1}\right|^{2} d x \leq K \int_{\Omega}\left|B^{*} \phi\right|^{2} d x .
$$

We define the set

$$
V_{h}^{1}(\tau)=\left\{\left.v\right|_{\tau}\left|v \in V_{h} ; L v\right|_{\sigma}=0 \forall \sigma \in \mathcal{E}_{v}\right\}
$$

and the linear functional

$$
f_{\tau}(\eta)=\int_{\tau}\left(B^{*} \phi\right) \eta d x
$$

for $\eta \in V_{h}^{1}(\tau)$. With the standard $L^{2}$ norm, $V_{h}^{1}(\tau)$ is a Hilbert space. By the Riesz representation theorem, there exist $v_{1, \tau} \in V_{h}^{1}(\tau)$ such that

$$
f_{\tau}(\eta)=\int_{\tau} v_{1, \tau} \eta d x \quad \text { and } \quad\left\|v_{1, \tau}\right\|_{L^{2}(\tau)}=\left\|f_{\tau}\right\|_{L^{2}(\tau)^{*}},
$$

where $*$ denotes a norm in the dual space. That is,

$$
\left\|f_{\tau}\right\|_{L^{2}(\tau)^{*}}=\sup _{\eta \in V_{h}^{1}(\tau)} \frac{f_{\tau}(\eta)}{\|\eta\|_{L^{2}(\tau)}} .
$$

We define $v_{1}$ such that $\left.v_{1}\right|_{\tau}=v_{1, \tau}$. Then

$$
\int_{\Omega}\left|v_{1}\right|^{2} d x=\sum_{\tau \in \mathcal{T}} \int_{\tau}\left|v_{1, \tau}\right|^{2} d x \leq \sum_{\tau \in \mathcal{T}} \int_{\tau}\left|B^{*} \phi\right|^{2} d x=\int_{\Omega}\left|B^{*} \phi\right|^{2} d x,
$$

which proves the second inequality in (5.8). To prove the first inequality in (5.8), we will first show $\left\|f_{\tau}\right\|_{L^{2}(\tau)^{*}}$ defines a norm for $B^{*} U_{h}$ on $\tau$. So, it suffices to show $B^{*} \phi=0$ if $\left\|f_{\tau}\right\|_{L^{2}(\tau)^{*}}=0$. Assume $\left\|f_{\tau}\right\|_{L^{2}(\tau)^{*}}=0$. Then we have $\int_{\tau}\left(B^{*} \phi\right) \eta d x=0$ for all $\eta \in V_{h}^{1}(\tau)$. Notice that $B^{*} \phi \in P^{k}(\tau)^{2}$. Taking $L \eta=0$ and $L^{\perp} \eta=L^{\perp}\left(B^{*} \phi\right)$ yields $\int_{\tau}\left(L^{\perp}\left(B^{*} \phi\right)\right)^{2} d x=0$. So, we have $L^{\perp}\left(B^{*} \phi\right)=0$ on $\tau$. By the definitions of $L^{\perp}$ and $B^{*}$, we have $\left(\partial_{\lambda_{\tau, 2}}-\partial_{\lambda_{\tau, 3}}\right) \phi=0$. So, we have

$$
\phi=\sum_{j=0}^{k+1} c_{j}\left(\lambda_{\tau, 2}+\lambda_{\tau, 3}\right)^{j} .
$$


Since $\phi \in U_{h}$, we have $c_{k+1}=0$ and therefore $L\left(B^{*} \phi\right) \in P^{k-1}(\tau)$. Taking $L^{\perp} \eta=0$ and $L \eta=\lambda_{\tau, 1} L\left(B^{*} \phi\right)$, we have $\int_{\tau} \lambda_{\tau, 1}\left(L\left(B^{*} \phi\right)\right)^{2} d x=0$, which implies $L\left(B^{*} \phi\right)=0$. Hence $B^{*} \phi=0$. Since norms in finite dimensional spaces are equivalent, we have $\left\|f_{\tau}\right\|_{L^{2}(\tau)^{*}} \geq K\left\|B^{*} \phi\right\|_{L^{2}(\tau)}$. Consequently, we obtain

$$
\int_{\Omega}\left(B^{*} \phi\right) v_{1} d x=\sum_{\tau \in \mathcal{T}} \int_{\tau}\left(B^{*} \phi\right) v_{1, \tau} d x=\sum_{\tau \in \mathcal{T}} f_{\tau}\left(v_{1, \tau}\right)=\sum_{\tau \in \mathcal{T}} \int_{\tau} v_{1, \tau}^{2} d x
$$

which proves the first inequality in (5.8).

We will find $v_{2} \in V_{h}$ and a function $v^{+}$such that

$$
\begin{aligned}
-\sum_{\sigma \in \mathcal{E}_{v}} \int_{\sigma}\left(L\left(v_{2}+v^{+}\right)\right)[\phi] d \sigma & \geq K_{2} \sum_{\sigma \in \mathcal{E}_{v}^{0}}\left(h_{\sigma}\right)^{-1} \int_{\sigma}[\phi]^{2} d \sigma, \\
\int_{\Omega}\left|v_{2}+v^{+}\right|^{2} d x & \leq K_{3} \sum_{\sigma \in \mathcal{E}_{v}^{0}}\left(h_{\sigma}\right)^{-1} \int_{\sigma}[\phi]^{2} d \sigma .
\end{aligned}
$$

Let $\sigma \in \mathcal{E}_{v}^{0}$ and let $\tau, \tilde{\tau}$ be the two triangles sharing the same edge $\sigma$. We define $V_{h}^{2}(\tau \cup \tilde{\tau})$ as follows:

$$
V_{h}^{2}(\tau \cup \tilde{\tau})=\left\{\left.v\right|_{\tau \cup \tilde{\tau}}+\nabla\left(\lambda_{\tau, 2} \lambda_{\tau, 3} q_{k}\right) \mid v \in V_{h},(V D 1)=(V D 2)=0 ; q_{k} \in P^{k}(\tau \cup \tilde{\tau})\right\} .
$$

Here, by $(\mathrm{VD} 1)=(\mathrm{VD} 2)=0$, we mean both the degrees of freedom defined by (VD1) and (VD2) are equal to zero. Also, the polynomial $q_{k}$ is fixed and will be chosen in the following. With the standard $L^{2}(\tau \cup \tilde{\tau})^{2}$ norm, $V_{h}^{2}(\tau \cup \tilde{\tau})$ is a Hilbert space. We also define the following linear functional:

$$
g_{\tau}(\eta)=\int_{\sigma}(L \eta)[\phi] d \sigma
$$

for all $\eta \in V_{h}^{2}(\tau \cup \tilde{\tau})$. By the Riesz representation theorem, there is an element $v_{2, \tau}+v_{\tau}^{+} \in V_{h}^{2}(\tau \cup \tilde{\tau})$ such that

$$
g_{\tau}(\eta)=\int_{\tau \cup \tilde{\tau}}\left(v_{2, \tau}+v_{\tau}^{+}\right) \eta d x \quad \text { and } \quad\left\|v_{2, \tau}+v_{\tau}^{+}\right\|_{L^{2}(\tau \cup \tilde{\tau})^{2}}=\left\|g_{\tau}\right\|_{\left(L^{2}(\tau \cup \tilde{\tau})^{2}\right)^{*}},
$$

where the norm $\left\|g_{\tau}\right\|_{\left(L^{2}(\tau \cup \tilde{\tau})^{2}\right)^{*}}$ is defined as

$$
\left\|g_{\tau}\right\|_{\left(L^{2}(\tau \cup \tilde{\tau})^{2}\right)^{*}}=\sup _{\eta \in V_{h}^{2}(\tau \cup \tilde{\tau})} \frac{g_{\tau}(\eta)}{\|\eta\|_{L^{2}(\tau \cup \tilde{\tau})^{2}}} .
$$

We then define $v_{2}$ by $\left.v_{2}\right|_{\tau}=v_{2, \tau}$ and $v^{+}$by $\left.v^{+}\right|_{\tau}=v_{\tau}^{+}$. Since $\int_{\sigma}(L \eta)^{2} d \sigma \leq K\left(h_{\sigma}^{-1}\right)$ $\int_{\tau \cup \tilde{\tau}}|\eta|^{2} d x$, we have

$$
\left\|g_{\tau}\right\|_{\left(L^{2}(\tau \cup \tilde{\tau})^{2}\right)^{*}}^{2} \leq \frac{1}{\|\eta\|_{L^{2}(\tau \cup \tilde{\tau})^{2}}^{2}} \int_{\sigma}(L \eta)^{2} d \sigma \int_{\sigma}[\phi]^{2} d \sigma \leq K h_{\sigma}^{-1} \int_{\sigma}[\phi]^{2} d \sigma .
$$

Summing up all $\tau \in \mathcal{T}$ proves (5.10). To prove (5.9), we will first show that $\left.[\phi]\right|_{\sigma}=$ 0 if $\left\|g_{\tau}\right\|_{\left(L^{2}(\tau \cup \tilde{\tau})^{2}\right)^{*}}=0$. Now, we assume $\left\|g_{\tau}\right\|_{\left(L^{2}(\tau \cup \tilde{\tau})^{2}\right)^{*}}=0$. Then we have 
$\int_{\sigma}(L \eta)[\phi] d \sigma=0$ for all $\eta \in V_{h}^{2}(\tau \cup \tilde{\tau})$. We take $\left.\eta\right|_{\tau} \in P^{k}(\tau)^{2}$ and $\left.\eta\right|_{\tilde{\tau}} \in P^{k}(\tilde{\tau})^{2}$ such that $L \eta=[\phi]$ at the $k+1$ Gaussian points of $\sigma$. Then, $\eta \in V_{h}^{2}(\tau \cup \tilde{\tau})$ and

$$
h_{\sigma} \sum_{j=1}^{k+1} w_{j}\left[\phi\left(\alpha_{j}\right)\right]^{2} d x=\int_{\sigma}(L \eta)[\phi] d x=0,
$$

where the first equality follows from the Gaussian quadrature rule; here $w_{j}$ denotes the weight and $\alpha_{j}$ denotes the quadrature point. Since the weights $w_{j}>0$, we have $[\phi]=0$ at the $k+1$ Gaussian points of $\sigma$. Notice that $\left.[\phi]\right|_{\sigma}$ is a polynomial of degree $k+1$. So, we have that $\left.[\phi]\right|_{\sigma}$ is a scalar multiple of the $(k+1)$ th degree Legendre polynomial, namely $\left.[\phi]\right|_{\sigma}=b \mathbb{P}_{k+1}$ for some constant $b$, where $\mathbb{P}_{k+1}$ is the Legendre polynomial of degree $k+1$. We take $\eta=\nabla\left(\lambda_{\tau, 2} \lambda_{\tau, 3} q_{k}\right)$ with $q_{k}$ to be determined below. Notice that $L \eta$ is the tangential derivative of $\lambda_{\tau, 2} \lambda_{\tau, 3} q_{k}$ along $\sigma$. By a change of variable, we have

$$
\begin{aligned}
\int_{\sigma}(L \eta)[\phi] d \sigma & =d \int_{-1}^{1} \frac{d}{d z}\left(\left(1-z^{2}\right) q_{k}(z)\right)[\phi(z)] d z \\
& =d \int_{-1}^{1} \frac{d}{d z}\left(\left(1-z^{2}\right) q_{k}(z)\right) b \mathbb{P}_{k+1}(z) d z
\end{aligned}
$$

for some constant $d>0$. Notice that $\mathbb{P}_{k+1}$ satisfies the Legendre differential equation

$$
\frac{d}{d z}\left(\left(1-z^{2}\right) \frac{d \mathbb{P}_{k+1}}{d z}\right)=-(k+1)(k+2) \mathbb{P}_{k+1} .
$$

So, we take $\left.q_{k}\right|_{\sigma}=\frac{d \mathbb{P}_{k+1}}{d z}$ and extend the definition of $q_{k}$ over all of $\tau \cup \tilde{\tau}$. Then we obtain

$$
\int_{\sigma}(L \eta)[\phi] d x=-b d(k+1)(k+2) \int_{-1}^{1} \mathbb{P}_{k+1}^{2}(z) d z
$$

This implies that $b=0$. Hence $[\phi]=0$. So, $\left\|g_{\tau}\right\|_{\left(L^{2}(\tau \cup \tilde{\tau})^{2}\right)^{*}}$ defines a norm on $L^{2}(\sigma)$. Since norms in finite dimensional spaces are equivalent, we obtain

$$
\left\|g_{\tau}\right\|_{\left(L^{2}(\tau \cup \tilde{\tau})^{2}\right)^{*}} \geq K(\tau \cup \tilde{\tau})\|[\phi]\|_{L^{2}(\sigma)} .
$$

A standard scale change argument yields

$$
\left\|g_{\tau}\right\|_{\left(L^{2}(\tau \cup \tilde{\tau})^{2}\right)^{*}} \geq K\left(h_{\sigma}\right)^{-\frac{1}{2}}\|[\phi]\|_{L^{2}(\sigma)},
$$

which proves (5.9).

Combining (5.8), (5.9), and (5.10), we have

$$
\begin{aligned}
B_{h}\left(\delta v_{1}+v_{2}+v^{+}, \phi\right) \geq & \delta K_{1} \int_{\Omega}\left|B^{*} \phi\right|^{2} d x+K_{2} \sum_{\sigma \in \mathcal{E}_{v}}\left(h_{\sigma}\right)^{-1} \int_{\sigma}[\phi]^{2} d \sigma \\
& +\int_{\Omega}\left(B^{*} \phi\right)\left(v_{2}+v^{+}\right) d x .
\end{aligned}
$$

By the Cauchy-Schwarz inequality,

$$
\int_{\Omega}\left(B^{*} \phi\right)\left(v_{2}+v^{+}\right) d x \geq-\frac{K_{2}}{2 K_{3}} \int_{\Omega}\left|v_{2}+v^{+}\right|^{2} d x-\frac{K_{3}}{2 K_{2}} \int_{\Omega}\left|B^{*} \phi\right|^{2} d x .
$$


So, we have

$$
B_{h}\left(\delta v_{1}+v_{2}+v^{+}, \phi\right) \geq\left(\delta K_{1}-\frac{K_{3}}{2 K_{2}}\right) \int_{\Omega}\left|B^{*} \phi\right|^{2} d x+\frac{K_{2}}{2} \sum_{\sigma \in \mathcal{E}_{v}}\left(h_{\sigma}\right)^{-1} \int_{\sigma}[\phi]^{2} d \sigma .
$$

Now, we choose $\delta$ such that $\delta K_{1}-\frac{K_{3}}{2 K_{2}}=1$. Then we have

$$
B_{h}\left(\delta v_{1}+v_{2}+v^{+}, \phi\right) \geq \min \left(1, \frac{K_{2}}{2}\right)\|\phi\|_{Z}^{2} .
$$

Since $B_{h}\left(v^{+}, \phi\right)=0$, we have

$$
B_{h}\left(\delta v_{1}+v_{2}, \phi\right) \geq \min \left(1, \frac{K_{2}}{2}\right)\|\phi\|_{Z}^{2} .
$$

We take $v=\delta v_{1}+v_{2} \in V_{h}$ so that the first inequality in (5.7) is proved. To prove the second inequality in (5.7), we first notice that $\left\|v_{2, \tau}\right\|_{L^{2}(\sigma)}$ defines a norm for $V_{h}^{2}(\tau \cup \tilde{\tau})$ since $\left\|v_{2, \tau}\right\|_{L^{2}(\sigma)}=0$ implies (VD3) $=0$ which in turn implies $v_{2, \tau}=0$ by unisolvence of the FE space $V_{h}$. By norm equivalence in finite dimensional spaces, we have $\left\|v_{2, \tau}\right\|_{L^{2}(\tau)} \leq K h^{\frac{1}{2}}\left\|v_{2, \tau}\right\|_{L^{2}(\sigma)} \leq\left\|v_{2, \tau}\right\|_{L^{2}(\tau)}$. Then we obtain

$$
\left\|\delta v_{1}+v_{2}\right\|_{L^{2}(\tau)} \leq \delta\left\|v_{1}\right\|_{L^{2}(\tau)}+\left\|v_{2}\right\|_{L^{2}(\tau)} \leq K\left\|v_{1}\right\|_{L^{2}(\tau)}+h^{\frac{1}{2}}\left\|v_{2}\right\|_{L^{2}(\sigma)} .
$$

Furthermore, we have the following orthogonality condition:

$$
\begin{aligned}
\int_{\sigma} v_{2, \tau} v_{\tau}^{+} d \sigma & =\int_{-1}^{1} v_{2, \tau} \frac{d}{d z}\left(\left(1-z^{2}\right) \frac{d \mathbb{P}_{k+1}}{d z}\right) d z \\
& =-(k+1)(k+2) \int_{-1}^{1} v_{2, \tau} \mathbb{P}_{k+1} d z=0
\end{aligned}
$$

since the function $\mathbb{P}_{k+1}$ is equal to zero at the $k+1$ Gaussian points of $\sigma$. By the orthogonality condition,

$$
\left\|v_{2}\right\|_{L^{2}(\sigma)}^{2} \leq\left\|v_{2}\right\|_{L^{2}(\sigma)}^{2}+\left\|v^{+}\right\|_{L^{2}(\sigma)}^{2}=\left\|v_{2}+v^{+}\right\|_{L^{2}(\sigma)}^{2} \leq K h^{-1}\left\|v_{2}+v^{+}\right\|_{L^{2}(\tau \cup \tilde{\tau})}^{2},
$$

where the last inequality follows from trace inequality. So, we have

$$
\left\|\delta v_{1}+v_{2}\right\|_{L^{2}(\tau \cup \tilde{\tau})^{2}} \leq K\left\|v_{1}\right\|_{L^{2}(\tau \cup \tilde{\tau})^{2}}+K\left\|v_{2}+v^{+}\right\|_{L^{2}(\tau \cup \tilde{\tau})^{2}} .
$$

Summing up all $\tau \in \mathcal{T}$ and using estimates (5.8) and (5.10) completes the proof.

6. Numerical examples. In this section we present a series of numerical experiments which give quantitative results and confirm the rate of convergence of the method (3.3)-(3.4). We will, in particular, consider the TE mode of Maxwell's equations (E) and set $\Omega=[0,2 \pi]^{2}, a_{1}=a_{2}=1$, and $F_{1}=F_{2}=0$. In addition, the function $u$ is the magnetic field $H$ while the vector $v$ is the electric field $E$. The exact solution to Maxwell's equations is

$$
\begin{aligned}
H(x, t) & =\cos (t) \cos \left(x_{1}\right)+\cos (t) \cos \left(x_{2}\right), \\
E_{1}(x, t) & =-\sin (t) \sin \left(x_{2}\right), \\
E_{2}(x, t) & =\sin (t) \sin \left(x_{1}\right) .
\end{aligned}
$$


TABLE 6.1

$L^{2}$ norm errors at $T=\pi / 4$ for $k=0$. Rate of convergence is 1.0298 .

\begin{tabular}{|c|c|c|}
\hline$N$ & $N T$ & $L^{2}$ error \\
\hline 10 & 100 & 1.311 \\
\hline 20 & 200 & 0.4799 \\
\hline 40 & 400 & 0.2782 \\
\hline 80 & 800 & 0.1301 \\
\hline 160 & 1600 & 0.06653 \\
\hline 320 & 3200 & 0.03378 \\
\hline
\end{tabular}

TABLE 6.2

Errors in various norms at $T=\pi / 4$ for $k=1$.

\begin{tabular}{|c|c|c|c|c|}
\hline$N$ & $N T$ & $L^{2}$ error & $\left\|H-H_{h}\right\|_{Z}$ & $\left\|E-E_{h}\right\|_{Z^{\prime}}$ \\
\hline 10 & 100 & 0.1809 & 1.526 & 0.7213 \\
\hline 20 & 200 & 0.04528 & 0.6619 & 0.3472 \\
\hline 40 & 400 & 0.01111 & 0.3498 & 0.1623 \\
\hline 80 & 800 & 0.002797 & 0.1597 & 0.1019 \\
\hline 160 & 1600 & 0.0007022 & 0.06220 & 0.04968 \\
\hline
\end{tabular}

The domain $\Omega$ is triangulated in the following manner. First, we divide $\Omega$ into $N \times N$ uniform squares. Then, we subdivide each square by connecting the lower left corner and the upper right corner to obtain two triangles. We further subdivide each triangle into three triangles by connecting the center of the triangle to its three vertices. For the resulting ODE system in time, we use the standard leap-frog scheme. Below we use NT to represent the number of time steps.

We first consider an example for the first order method, that is, $k=0$. We then test the rate of convergence by comparing the solution to the scheme (3.3)-(3.4) and the exact solution at $T=\frac{\pi}{4}$. Table 6.1 shows the discrete $L^{2}$ errors for various mesh sizes, from $N=10$ to $N=320$. Here, we choose the time step small enough so that a suitable CFL condition for the leap-frog scheme is satisfied. We will use the results from Table 6.1 and the least squares method to estimate the rates of convergence of the scheme in the discrete $L^{2}$ norm. More precisely, we assume the error is proportional to $h^{\beta}$ for some $\beta \in \mathbb{R}$, and then perform a least square data fitting using the data from Table 6.1. Doing this, the numerical rate of convergence is 1.0298. This confirms that the scheme is first order convergence in the discrete $L^{2}$ norm.

Next, we consider an example with $k=1$, that is, the FE spaces $U_{h}$ and $V_{h}$, which contain all linear polynomials and a subset of quadratic polynomials. We will test the rates of convergence in various norms at $T=\pi / 4$. Table 6.2 shows the results of error in various norms with various mesh sizes. In the third column of Table 6.2, we give the sum of the error for both $H$ and $E$ in the discrete $L^{2}$ norm. In the fourth column, we have the error for the magnetic field in the $Z$-norm. In the fifth column, we have the error for the electric field in the $Z^{\prime}$-norm. Table 6.3 shows the estimated rates of convergence. From the table, we see that the estimated rate of convergence in the discrete $L^{2}$ norm is approximately 2. Moreover, the estimated rates of convergence in $H^{1}$ semi-norms are approximately equal to 1 . Our theoretical statements are thus confirmed by this experiment.

In Table 6.4, we also show the error for the divergence of $E$ as well as the normal jump of $E$. We have not proved convergence results in these two norms, but they are implied by the estimates that we proved in previous sections. The error in the 
TABLE 6.3

Estimated rate of convergence at $T=\pi / 4$ for $k=1$.

\begin{tabular}{|c|c|}
\hline Norm & Estimated rate \\
\hline$L^{2}$ norm & 2.004 \\
\hline$\left\|H-H_{h}\right\|_{Z}$ & 1.129 \\
\hline$\left\|E-E_{h}\right\|_{Z^{\prime}}$ & 0.9489 \\
\hline
\end{tabular}

TABLE 6.4

Normal jump and divergence errors at $T=\pi / 4$ for $k=1$.

\begin{tabular}{|c|c|c|c|}
\hline$N$ & $N T$ & $\sum_{\sigma \in \mathcal{E}}\left(h_{\sigma}^{-\frac{1}{2}}\right)\left\|L^{\perp}\left(E-E_{h}\right)\right\|_{L^{2}(\sigma)}$ & $\left\|\operatorname{div}\left(E-E_{h}\right)\right\|_{L^{2}(\Omega)}$ \\
\hline 10 & 100 & 1.212 & 0.2519 \\
\hline 20 & 200 & 0.5692 & 0.1180 \\
\hline 40 & 400 & 0.2610 & 0.05039 \\
\hline 80 & 800 & 0.1387 & 0.03494 \\
\hline 160 & 1600 & 0.06686 & 0.01632 \\
\hline
\end{tabular}

divergence of $E$ is measured by $\left\|\operatorname{div}\left(E-E_{h}\right)\right\|_{L^{2}(\Omega)}$ while the error in the normal jump of $E$ is measured by $\sum_{\sigma \in \mathcal{E}}\left(h_{\sigma}^{-\frac{1}{2}}\right)\left\|L^{\perp}\left(E-E_{h}\right)\right\|_{L^{2}(\sigma)}$. The estimated rates in both norms are 0.9652 and 1.040 , respectively. So, the rates of convergence are indeed first order for these two norms.

In what follows, we will consider the one dimensional scalar wave equation on $\Omega=[0,2 \pi]$

$$
\frac{\partial u}{\partial t}=\frac{\partial v}{\partial x}, \quad \frac{\partial v}{\partial t}=\frac{\partial u}{\partial x}
$$

with periodic boundary condition. The purpose is to compare our new optimal DG with the central DG method. The central DG method is typically based on piecewise polynomial approximation without continuity requirement across cell interfaces. Flux integrals along cell boundaries are evaluated by using the average of two values of the numerical solutions from the two neighboring cells, or the so called central numerical flux; see, for example, [7]. We choose $u(x, t)=e^{\sin (x-t)}$ and $v(x, t)=-e^{\sin (x-t)}$ to be the exact solution. We will compare the numerical solutions by the two methods at $T=20$ using 20 spatial cells and the leap-frog scheme for the time discretization. Figure 6.1 shows the numerical solutions. First, we see that both methods preserve energy. Second, we see that there are spurious modes in the numerical solutions obtained by the central DG. It can be shown that the central DG does not satisfy the inf-sup conditions that we introduce in this paper. With our new optimal DG, which verifies the inf-sup conditions, we see that there is no spurious mode appearing in the numerical solution.

Now we will compare our new DG with an upwind DG method. The upwind DG method is typically based on piecewise polynomial approximation without continuity requirement. Flux integrals along cell boundaries are evaluated by taking the upwind value of the numerical solution from the two neighboring cells, or the so called upwind numerical flux; see, for example, [10]. We will compare the numerical solutions by both methods using the same setting except that $T=100$ and the 4 th order RungeKutta method is used for time stepping for the upwind DG. Figure 6.2 shows the numerical results. We see that both methods contain no spurious mode. For the 


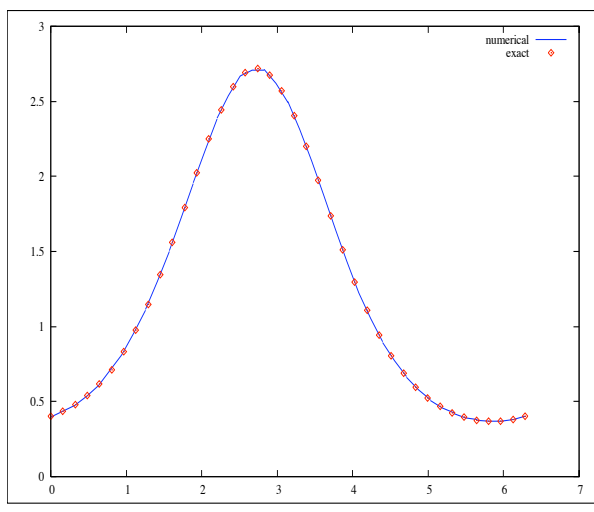

Our DG

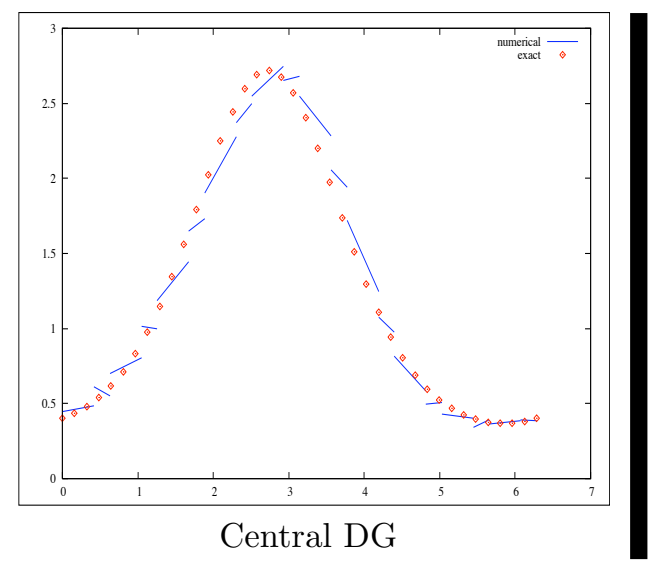

FIG. 6.1. Comparison of the optimal DGM and central DGM.

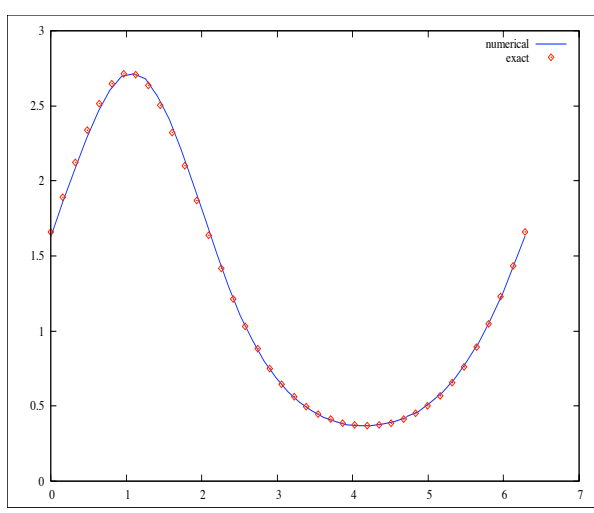

Our DG

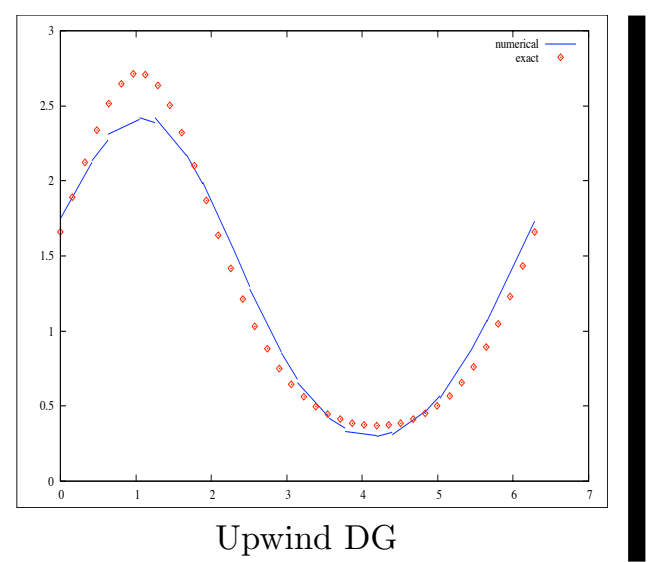

Upwind DG

FIG. 6.2. Comparison of the optimal DGM and upwind DGM.

upwind DG, it is well known that it is dissipative as seen from the numerical result. On the contrary, our optimal DG preserves energy well.

Before ending this section, we will compare the upwind DG, central DG, and our new optimal DG with piecewise linear approximation. Due to the nature of the three schemes, they are all explicit and suitable for unstructured grids. Because of upwinding, the upwind DG is not energy preserving. In terms of the total number of degrees of freedom (DOF), both the upwind DG and the central DG need $4 \mathrm{~N}$ unknowns. This is because there are 4 unknowns on each cell. On the contrary, owing to the extra continuity conditions, our new DG needs only $3 N$ unknowns, which is more efficient in terms of memory storage. In addition, the central DG is only first order accurate, which is suboptimal since we are considering piecewise linear approximation. Both the upwind DG and our new DG have optimal order of convergence, namely, second order in the $L^{2}$ norm. We summarize all these properties in Table 6.5.

7. Conclusion. In this paper, we have developed and analyzed a new class of discontinuous Galerkin methods. This new DG can be seen as a compromise between 
TABLE 6.5

Comparison among upwind, central, and our new DG with piecewise linear polynomials.

\begin{tabular}{|c|c|c|c|}
\hline & Upwind & Central & Our \\
\hline Explicit scheme & $\mathrm{Y}$ & $\mathrm{Y}$ & $\mathrm{Y}$ \\
\hline Unstructured grid & $\mathrm{Y}$ & $\mathrm{Y}$ & $\mathrm{Y}$ \\
\hline Energy conservation & $\mathrm{N}$ & $\mathrm{Y}$ & $\mathrm{Y}$ \\
\hline DOF & $4 N$ & $4 N$ & $3 N$ \\
\hline Order & $O\left(h^{2}\right)$ & $O(h)$ & $O\left(h^{2}\right)$ \\
\hline
\end{tabular}

the standard DG and the FE in the sense that our new DG is explicit as standard DG and is energy conserving as FE. Energy conservation is an important property for a large class of applications that involves the numerical solutions of wave equations while explicitness provides a more efficient scheme where no matrix inversion is needed at each time step. We have shown that the new DG is stable in both the discrete $L^{2}$ norm and discrete $H^{1}$ norm. Moreover, the convergence rate is optimal with respect to the order of the polynomial space. To the best of our knowledge, our new DG is the first method that satisfies all of the following properties: explicit, energy conserving, suitable for unstructured grids, and optimal rate of convergence.

\section{REFERENCES}

[1] E. Bécache, P. Joly, and C. Tsogka, An analysis of new mixed finite elements for the approximation of wave propagation problems, SIAM J. Numer. Anal., 37 (2000), pp. 10531084.

[2] F. Brezzi And M. Fortin, Mixed and hybrid finite element methods, Springer-Verlag, New York, 1991.

[3] P. G. Ciarlet, The Finite Element Method for Elliptic Problems, North-Holland Publishing, Amsterdam, 1978.

[4] B. Cockburn, F. Li, And C.-W. Shu, Local divergence-free discontinuous Galerkin methods for the Maxwell equations, J. Comput. Phys., 194 (2004), pp. 588-610.

[5] G. Cohen, P. Joly, N. Torjman, And J. Roberts, Higher order triangular finite elements with mass lumping for the wave equation, SIAM J. Numer. Anal., 38 (2001), pp. 2047-2078.

[6] G. Cohen and P. Monk, Gauss point mass lumping schemes for Maxwell's equations, Numer. Methods Partial Differential Equations, 14 (1998), pp. 63-88.

[7] L. Fezoui, S. Lanteri, S. Lohrengel, And S. Piperno, Convergence and stability of a discontinuous Galerkin time-domain method for the $3 D$ heterogeneous Maxwell equations on unstructured meshes, M2AN, Math. Model Numer. Anal., 39 (2005), pp. 1149-1176.

[8] T. GevecI, On the application of mixed finite element methods to the wave equations, RAIRO Modél. Math. Anal. Numér., 22 (1988), pp. 243-250.

[9] M. Grote, A. Schneebeli, And D. Schötzau, Discontinuous Galerkin finite element method for the wave equation, submitted.

[10] J. S. Hesthaven and T. Warburton, High-order nodal methods on unstructured grids, I. Time-domain solution of Maxwell's equations, J. Comput. Phys., 181 (2002), pp. 186-221.

[11] P. Joly, Variational methods for time-dependent wave propagation problems, Topics in Computational Wave Propagation, Lect. Notes Comput. Sci. Eng. 31, Springer-Verlag, Berlin, pp. 201-264.

[12] P. Monk, A mixed method for approximating Maxwell's equations, SIAM J. Numer. Anal., 28 (1991), pp. 1610-1634.

[13] P. Monk, An analysis of Nédélec's method for the spatial discretization of Maxwell's equations, J. Comput. Appl. Math., 47 (1993), pp. 101-121.

[14] P. Monk ANd G. R. Richter, A discontinuous Galerkin method for linear symmetric hyperbolic systems in inhomogeneous media, J. Sci. Comput., 22 (2005), pp. 443-477.

[15] J. NÉDÉLEC, Mixed finite elements in $\mathbb{R}^{3}$, Numer. Math., 35 (1980), pp. 315-341.

[16] K. S. YeE, Numerical solution of initial boundary value problems involving Maxwell's equations in isotropic media, IEEE Trans. Antennas Propagat., 14 (1966), pp. 302-307. 